\title{
Self-Concatenated Code Design and its Application in Power-Efficient Cooperative Communications
}

\author{
Muhammad Fasih Uddin Butt, Member, IEEE, Soon Xin Ng, Senior Member, IEEE, and \\ Lajos Hanzo, Fellow, IEEE
}

\begin{abstract}
In this tutorial, we have focused on the design of binary self-concatenated coding schemes with the help of EXtrinsic Information Transfer (EXIT) charts and Union bound analysis. The design methodology of future iteratively decoded self-concatenated aided cooperative communication schemes is presented. In doing so, we will identify the most important milestones in the area of channel coding, concatenated coding schemes and cooperative communication systems till date and suggest future research directions.
\end{abstract}

Index Terms - Near-Capacity Code Design, Self-Concatenated Convolutional Codes, SECCC, EXIT charts, Iterative Decoding, Cooperation Diversity, Distributed Coding.

\section{INTRODUCTION}

$\mathbf{T}$ HE NEED for high-rate wireless communication systems designed for supporting broadband wireless Internet and multimedia services has been growing over the past decade. However, the available radio spectrum is limited and the wireless channel is extremely hostile. Therefore, there is a demand for flexible and bandwidth-efficient transceivers [1], [2]. Shannon quantified the capacity of wireless communication systems in 1948 [3]. Advances in coding have made it feasible to approach Shannon's capacity limit for the case of a singleuser system [4], [5]. Multiple-Input Multiple-Output (MIMO) communication systems create multiple wireless links by employing multiple transmit and receive antennas, hence they are capable of supporting high-integrity, high data rate communications [6]. However, MIMOs cannot be readily implemented in shirt-pocket-sized mobile stations (MS), which hence have a limited antenna spacing and impose correlation of the signals. Cooperative communications is capable of eliminating this correlation, while still achieving MIMO-like diversity gains for the system [7]. This is achieved by introducing a relay between the source and the destination with the aid of an independently faded path created by the relay. Coded

Manuscript received 2 August 2010; revised 19 April 2011. The financial support of CIIT under the auspices of Higher Education Commission, Pakistan, and that of the EU Optimix Project of the RC-UK under the auspicies of the IU-ATC, as well as of Dr. Wali Mohammad Trust, is gratefully acknowledged. The authors would like to thank the anonymous reviewers for their valuable comments, which greatly improved the presentation of the paper.

M. F. U. Butt is with the Department of Electrical Engineering, COMSATS Institute of Information Technology (CIIT), Islamabad 44000, Pakistan (e-mail: fasih@comsats.edu.pk).

S. X. Ng and L. Hanzo are with the School of Electronics and Computer Science, University of Southampton, SO17 1BJ, Southampton, U.K. (e-mail: $\{$ sxn, lh\}@ecs.soton.ac.uk).

Digital Object Identifier 10.1109/SURV.2011.081511.00104 cooperation [8] is potentially capable of flawlessly recovering the original source signal at the relays and then retransmitting it to the destination from a reduced distance.

In this tutorial, we have presented a brief history of channel coding and then highlighted the differences between iteratively decoded Parallel Concatenated Convolutional Coding (PCCC), Serial Concatenated Convolutional Coding (SCCC) and SelfConcatenated Convolutional Coding (SECCC) schemes. We then explored SECCC schemes that are designed for transmission over Additive White Gaussian Noise (AWGN) and uncorrelated Rayleigh fading channels. We designed both bit-based SECCC and SECCC employing Iterative Decoding (SECCC-ID), using a Recursive Systematic Convolutional (RSC) constituent encoder. On the other hand, Low Density Parity Check (LDPC) codes constitute another attractive code family which can be described based on the sparse-graph [9][11]. It was shown in [9] that it is possible to describe the PCCC schemes using the sparse-graph as well. However, we only consider trellis-based decoding in this paper.

EXtrinsic Information Transfer (EXIT) charts were used as our main design tools. We will exemplify the proposed design procedures and demonstrate that some of the proposed schemes are capable of operating within about $1 \mathrm{~dB}$ from the AWGN and Rayleigh fading channels' capacity. The union bound analysis of SECCCs was carried out for finding the corresponding Bit Error Ratio (BER) floors. In order to further exploit the benefits of the low complexity design offered by SECCCs we explored their application in a distributed coding scheme designed for cooperative communications, where iterative detection is employed by exchanging extrinsic information between the decoders of SECCC and RSC at the destination. It was shown that the DSECCC-ID is a lowcomplexity scheme, yet capable of approaching the Discreteinput Continuous-output Memoryless Channels's (DCMC) capacity. Our discussions demonstrate that the proposed scheme is capable of reliably operating at a low BER for transmission over uncorrelated Rayleigh fading channels. In our cooperative communication schemes considered we assume that decoding errors may be encountered at the relay nodes and successfully mitigate their effects.

The outline of the paper is as follows. Section II discusses iterative detection aided coded modulation schemes designed for transmission over non-dispersive propagation environments. SECCC and SECCC-ID schemes using iterative detection are designed with the aid of EXIT charts in 


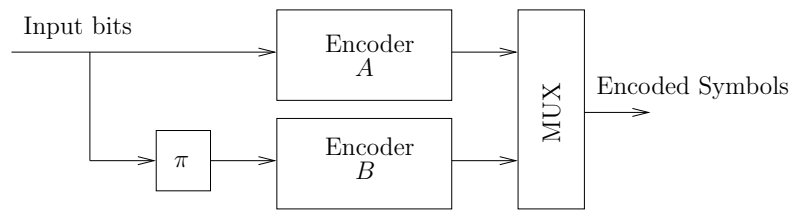

(a) PCCC Encoder

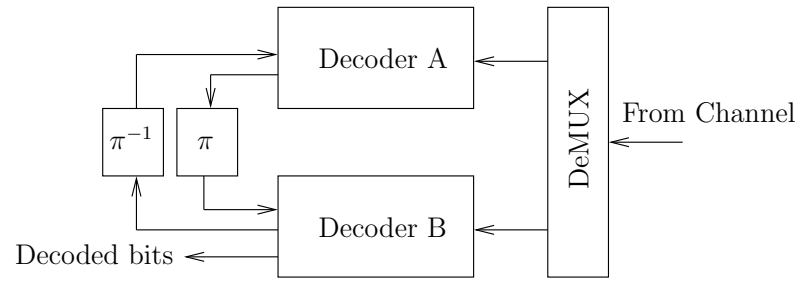

(b) PCCC decoder

Fig. 1. The schematic of a PCCC encoder and decoder.

Section III. In order to mitigate the effects of large-scale shadow fading on the performance of wireless communication systems, we present a distributed coding scheme in Section IV for cooperative communications employing SECCCs that is capable of providing substantial diversity-, throughput- as well as coding-gains for a single-user scenario. Finally, in Section $\mathrm{V}$ the main findings of the paper are summarised, general design guidelines are presented and future research directions are discussed.

\section{Iterative Decoding AND CONVERGEnCE ANALysis OF CONCATENATED CODES}

Forward Error Correction (FEC) or Channel Coding in the context of digital communication has a history dating back to the middle of the twentieth century. In recent years, the field has been revolutionized by iterative detection aided codes, which are capable of approaching the theoretical limits of performance, namely the channel capacity. Important milestones in the area of channel coding are described in Table I.

When the concatenated coding philosophy [25] was conceived back in 1966, it was deemed to have an excessive complexity and hence the resultant codes failed to stimulate immediate research interests. It was not until the discovery of Turbo Codes (TC) by Berrou et al. in 1993 [4], that efficient iterative decoding of concatenated codes became a reality at a low complexity by employing low-complexity constituent codes. There are three major types of iteratively decoded concatenated coding schemes, as discussed below:

\section{A. Parallel Concatenated Convolutional Codes}

Classic TCs [4] consist of two or more parallel constituent codes [80]. The component codes are usually systematic codes, because their systematic nature simplifies the iterative exchange of information between the constituent decoders. In general, each component encoder independently encodes its input information and an interleaver $(\pi)$ - also often termed as a scrambler - is used between the two constituent encoders to make both their input data and their encoded data statistically independent of each other, as shown in Fig. 1(a).

Again, the encoders used in classic TCs are almost always RSC encoders, which output both the original information
TABLE I

MiLESTONES IN CHANNEL CODING (1948-2008)

\begin{tabular}{|c|c|}
\hline Year & Milestone \\
\hline$\overline{1948}$ & Shannon's Capacity Theorem [3]. \\
\hline 1950 & Hamming codes were discovered by Hamming [12]. \\
\hline 1954 & Reed [13] and Muller [14] present Reed-Muller (RM) codes. \\
\hline 1955 & Elias introduces convolutional codes [15]. \\
\hline 1957 & Prange introduces cyclic codes [16]. \\
\hline 1959 & Hocquenghem [17] and ... \\
\hline 1960 & $\begin{array}{l}\text { Bose and Chaudhuri [18] proposed BCH codes. } \\
\text { Reed and Solomon defined (RS) codes over certain finite Galois fields [19]. } \\
\text { Peterson designed a BCH decoder [20]. }\end{array}$ \\
\hline 1961 & Peterson's book on Error Correction Codes (ECC) [21]. \\
\hline 1962 & Gallager invents LDPC codes [22]. \\
\hline 1963 & $\begin{array}{l}\text { Fano algorithm introduced for decoding convolutional codes [23]. } \\
\text { Massey describes threshold decoding [24]. }\end{array}$ \\
\hline 1966 & $\begin{array}{l}\text { Forney's introduction of concatenated codes [25] } \\
\text { and generalized minimum distance decoding [26]. }\end{array}$ \\
\hline 1967 & $\begin{array}{l}\text { Berlekamp designs an efficient algorithm for BCH/RS decoding [27]. } \\
\text { Rudolph initiates the study of finite geometries for coding [28]. }\end{array}$ \\
\hline 1968 & $\begin{array}{l}\text { Berlekamp, documents Algebraic Coding Theory [29]. } \\
\text { Gallager publishes, Information theory and reliable communications [30]. }\end{array}$ \\
\hline 1969 & $\begin{array}{l}\text { Jelinek defines the stack algorithm for decoding convolutional codes [31]. } \\
\text { Massey introduces his BCH decoding algorithm [32]. } \\
\text { Reed-Muller code used on Mariner deep space probes. }\end{array}$ \\
\hline 1971 & $\begin{array}{l}\text { Viterbi algorithm for Maximum Likelihood (ML) decoding of convolutional } \\
\text { codes [33]. }\end{array}$ \\
\hline 1972 & $\begin{array}{l}\text { Bahl et al. invents the Maximum A-Posteriori (MAP) algorithm [34]. } \\
\text { Chase introduces his soft-decision-based block decoding algorithm [35]. } \\
\text { Peterson and Weldon revise their book [36]. }\end{array}$ \\
\hline 1973 & Forney further interprets the Viterbi algorithm [37]. \\
\hline 1974 & Bahl et al. describe the symbol based MAP algorithm [38]. \\
\hline 1975 & Sugiyama et al. invokes the Euclidean algorithm for decoding [39]. \\
\hline 1977 & $\begin{array}{l}\text { MacWilliams and Sloane write The Theory of Error Correcting Codes [40]. } \\
\text { Voyager deep space mission uses a concatenated RS/convolutional code } \\
\text { (see [41]). }\end{array}$ \\
\hline 1978 & Wolf introduces trellis-decoding of block codes [42]. \\
\hline 1980 & $\begin{array}{l}\text { Sony and Phillips standardize the compact disc, including } \\
\text { a shortened RS code. }\end{array}$ \\
\hline 1981 & Goppa introduces Algebraic-Geometry (AG) codes [43], [44]. \\
\hline 1982 & Ungerböck invents trellis-coded modulation (TCM) [45]. \\
\hline 1983 & $\begin{array}{l}\text { Textbook on Error control coding by Lin and Costello [46]. } \\
\text { Blahut publishes his channel coding book [47]. }\end{array}$ \\
\hline 1988 & Divsalar and Simon discover multiple trellis-coded modulation [48]. \\
\hline 1989 & Hagenauer and Hoeher present the Soft-Output Viterbi Algorithm (SOVA) [49]. \\
\hline 1990 & Koch and Baier describe a reduced complexity MAP algorithm [50]. \\
\hline 1992 & Zehavi introduces Bit-Interleaved Coded Modulation (BICM) [51]. \\
\hline 1993 & $\begin{array}{l}\text { Berrou, Glavieux, and Thitimajshima discover turbo codes [4]. } \\
\text { Honary, Markarian and Farrell et al. presented low complexity trellis } \\
\text { decoding } \\
\text { of array [52] and Hamming codes [53]. }\end{array}$ \\
\hline 1994 & $\begin{array}{l}\text { The } Z_{4} \text { linearity of certain families of nonlinear codes is announced [54]. } \\
\text { Erfanian, Pasupathy and Gulak describe the Max-log-MAP algorithm [55]. }\end{array}$ \\
\hline 1995 & $\begin{array}{l}\text { MacKay revives LDPC codes [56]. } \\
\text { Wicker publishes his textbook [57]. } \\
\text { Robertson, Villebrun and Hoeher desribe Log-MAP algorithm [58]. }\end{array}$ \\
\hline 1996 & $\begin{array}{l}\text { Hagenauer, Offer and Papke propose turbo-BCH codes [59]. } \\
\text { Sidorenko, Markarian and Honary presented a novel trellis design technique } \\
\text { [60] for block and convolutional codes resulting in low complexity Viterbi } \\
\text { decoding. }\end{array}$ \\
\hline 1997 & $\begin{array}{l}\text { Tarokh, Seshadri and Calderbank introduce space-time trellis coding (STTC) } \\
\text { scheme [61]. } \\
\text { Nickl, Hagenauer and Burkett report approaching the Shannon limit over } \\
\text { Gaussian channels [62] within } 0.27 \mathrm{~dB} \text {. } \\
\text { Schlegel writes his book on trellis coding [63]. } \\
\text { Ritcey and Li introduce Bit-Interleaved Coded Modulation with Iterative } \\
\text { Decoding (BICM-ID) [64]. }\end{array}$ \\
\hline 1998 & $\begin{array}{l}\text { Turbo trellis-coded modulation (TTCM) introduced by Robertson and Wörz [65]. } \\
\text { Alamouti introduces space-time block coding [66]. } \\
\text { Guruswami and Sudan present a list decoder for RS and AG codes [67]. }\end{array}$ \\
\hline 1999 & Ritcey and Li combine TCM with BICM-ID [68]. \\
\hline 2000 & $\begin{array}{l}\text { Aji and McEliece [69] (and others [70]) synthesize } \\
\text { several decoding algorithms using message passing ideas. } \\
\text { Proakis publishes fourth edition of his textbook [71]. }\end{array}$ \\
\hline 2002 & $\begin{array}{l}\text { Hanzo, Liew, and Yeap characterize turbo algorithms in [5]. } \\
\text { Siwamogsatham and Fitz introduce MTCM assisted STBC [72]. }\end{array}$ \\
\hline 2003 & $\begin{array}{l}\text { Jafarkhani and Seshadri propose super-orthogonal STTC (SOSTTC) [73]. } \\
\text { Koetter and Vardy extend the GS algorithm for soft-decision } \\
\text { decoding of RS codes [74]. }\end{array}$ \\
\hline 2004 & Lin and Costello publish second edition of their textbook [75]. \\
\hline 2005 & $\begin{array}{l}\text { Moon publishes his textbook [76]. } \\
\text { Simon and Alouini write Digital Communications over Fading Channels [77]. } \\
\text { Song et al. introduce SOSTTC combined with QAM [78]. }\end{array}$ \\
\hline 2008 & $\begin{array}{l}\text { Arıan [79] introduce capacity-achieving Polar codes which are a extension } \\
\text { of RM codes for symmetric binary-input discrete memoryless channels. }\end{array}$ \\
\hline
\end{tabular}




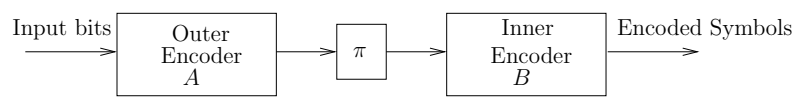

(a) SCCC Encoder

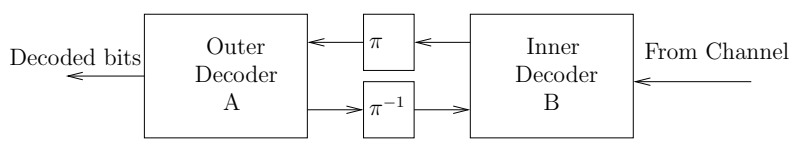

(b) SCCC decoder

Fig. 2. The schematic of an SCCC encoder and decoder.

bits that are also referred to as systematic bits and the corresponding parity bits. Hence two codewords are generated by the two RSC codes, both of which contain the same original information bits, but typically these bits are only transmitted from one of the output streams. If both RSC encoders are half-rate encoders, the resultant TC becomes a third-rate code. However, the number of parity bits transmitted from the two streams can be appropriately adjusted by simply discarding the required fraction of parity bits. This so-called puncturing operation tacitly assumes that these bits were set to zero and hence the corresponding zeros have to be inserted in the right bit-positions at the decoder's input. In a nutshell, the redundant parity bits of both encoders may be transmitted, plus a single copy of the systematic information bit. At the decoder shown in Fig. 1(b), two RSC decoders are used, which iteratively exchange their so-called soft-information, before making a hard-decision after a sufficiently high number of iterations.

The RSC constituent codes of classic TCs may also be replaced by other constituent codes. Inspired by this turbo coding concept various other coding arrangements, such as Turbo Trellis Coded Modulation (TTCM) schemes were proposed in [81], [82] and [65], which have a similar architecture to classic TCs, but employ Trellis Coded Modulation (TCM) constituent codes [83]. The appealing philosophy of TCM schemes is that they combine channel coding and modulation in an ingenious way, where the modulated signal constellation is extended to an increased number of constellation points, so that more bits per symbol can be transmitted for the sake of absorbing the parity bits. This way the constellation points have a reduced Eucledian distance amongst them, which potentially results in an increased Bit Error Ratio (BER), but this is more than compensated by the error correction capability of the Forward Error Correction (FEC) codec. It was also shown by Robertson et al. in [65] that TTCM is capable of outperforming classic TC.

\section{B. Serial Concatenated Convolutional Codes}

The serial concatenation of an outer and an inner encoder is shown in Fig. 2(a). These codes were discovered by Benedetto et al. [84]. Typically the inner code is a weaker code and the outer code is a stronger code, which are separated by an interleaver as shown in Fig. 2(a). The SCCCC decoder is shown in Fig. 2(b).

To obtain higher code rates we may employ puncturing. SCCC codes have been shown to yield a performance comparable, and in some cases superior, to TC.

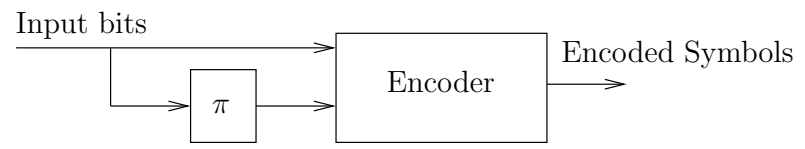

(a) SECCC Encoder

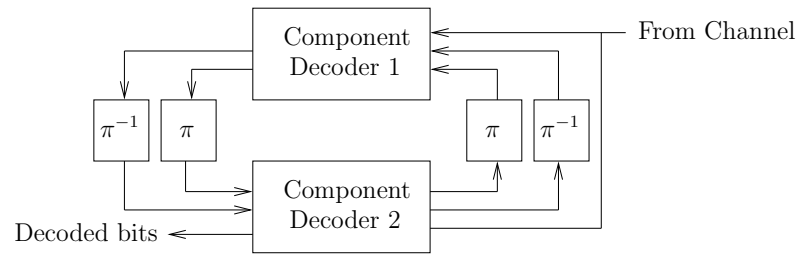

(b) SECCC decoder

Fig. 3. The schematic of an SECCC encoder and decoder.

\section{Self-Concatenated Convolutional Codes}

Self-concatenated convolutional codes (SECCC) for BPSK modulation were proposed by [85], [86]. SECCC is similar to PCCC when two component codes are replaced by one component code employing an odd-even separated turbo interleaver as discovered in [87]. SECCCs exhibit a low complexity, since they invoke only a single encoder as depicted in Fig. 3(a) and a single decoder as shown in Fig. 3(b).

Iterative decoding works by exchanging extrinsic information between the component decoders 1 and 2. The soft extrinsic information of one decoder is fed to the other constituent decoder as its a priori input which improves its knowledge and hence performance. The decoders iterate until there is no improvement achieved from the feedback and at that point correct decoding of the bits is possible. This point is called the convergence point. Iteratively-Decoded SelfConcatenated Trellis Coded Modulation (SECTCM) schemes for higher modulation were proposed by Benedetto et al. [88] and Loeliger [89]. It can be seen from Fig. 4 that the performance of the SECTCM code improves by increasing the number of self-iterations, hence exhibiting a turbolike behaviour for the case of uncorrelated Rayleigh fading channels. Since the pioneering work by Berrou et al. [4], the appealing iterative decoding of concatenated codes has inspired numerous researchers to extend the technique to other transmission schemes consisting of a concatenation of two or more constituent decoding stages.

The concept of EXIT charts was proposed by ten Brink in [90], [91] as a tool designed for analysing the convergence behaviour of iteratively decoded systems. Their attractive properties are listed below:

- EXIT charts constitute an efficient tool created for independently analysing each component of an iterative system.

- Amongst their other benefits detailed in Sections III-A1, III-B2 and IV-D they are capable of predicting the specific SNR value, where an infinitesimally low BER can be achieved without performing time-consuming bit-by-bit decoding employing a high number of iterations of the actual system.

- They analyse the input/output mutual information characteristics of a Soft-Input-Soft-Output (SISO) constituent 


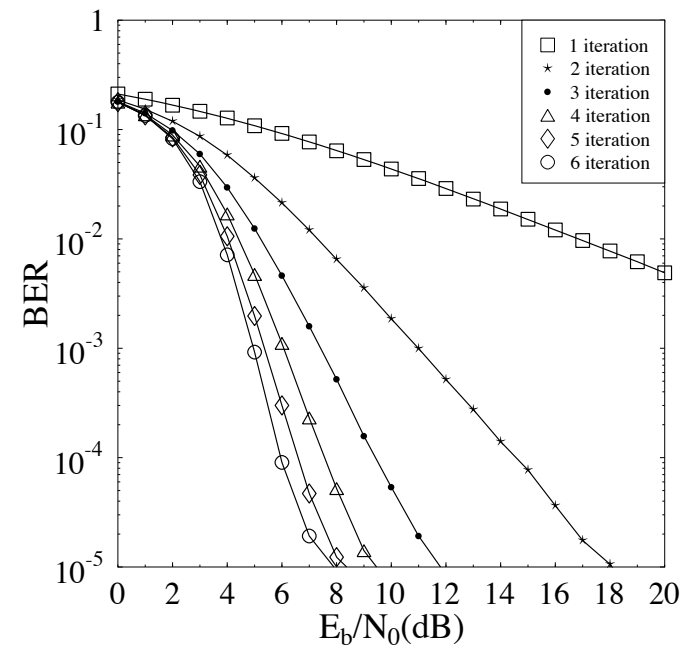

Fig. 4. Simulations results for 8 -state, rate-1/2 SECTCM code, when communicating over uncorrelated Rayleigh fading channels.

decoder by modelling the a priori Log-Likelihood Ratio (LLR) values and computing the corresponding mutual information between the hard-decision based bits and the extrinsic LLRs.

- The SNR value, where a 'waterfall-like' decay of the BER curve, called turbo-cliff [4], is observed for a concatenated code may be successfully predicted with the aid of EXIT charts.

- The SNR-distance from capacity is commensurate with the area of the open EXIT-chart tunnel, hence near capacity designs exhibit a marginally open tunnel. This typically imposes a high complexity associated with a high number of iterations and a long interleaver delay.

- If the Monte-Carlo simulation based stair-case shaped decoding trajectory reaches the $(1,1)$ point in the EXITchart, a vanishingly low BER may be achieved.

However, the EXIT chart based BER performance-prediction accuracy erodes, unless we assume the employment of a sufficiently long interleaver, so that the extrinsic LLRs can be rendered Gaussian distributed.

The EXIT chart analysis of the SECTCM decoder of [92] is shown in Fig. 5, which allows us to determine the number of self-iterations required by the SECTCM decoder to achieve convergence. The reason for the EXIT chart mismatch in Fig. 5 will be explained in Section III. An EXIT chart comprises of two EXIT curves for the two component decoders in the system, as shown in Fig. 5. Each curve plots the mutual information of the extrinsic LLRs versus the mutual information of the a priori LLRs of one decoder in the system, which is basically to measure the quality of the input and the output of the decoder. In order to achieve a vanishingly low BER at a specific $E_{b} / N_{0}$ value, component decoders' EXIT curves should only intersect at the $\left(I_{A}, I_{E}\right)=(2,2)$ point of the EXIT chart for the case of Symbol-based SECTCM (Fig. 5) and $\left(I_{A}, I_{E}\right)=(1,1)$ point for bit-based schemes of Section III

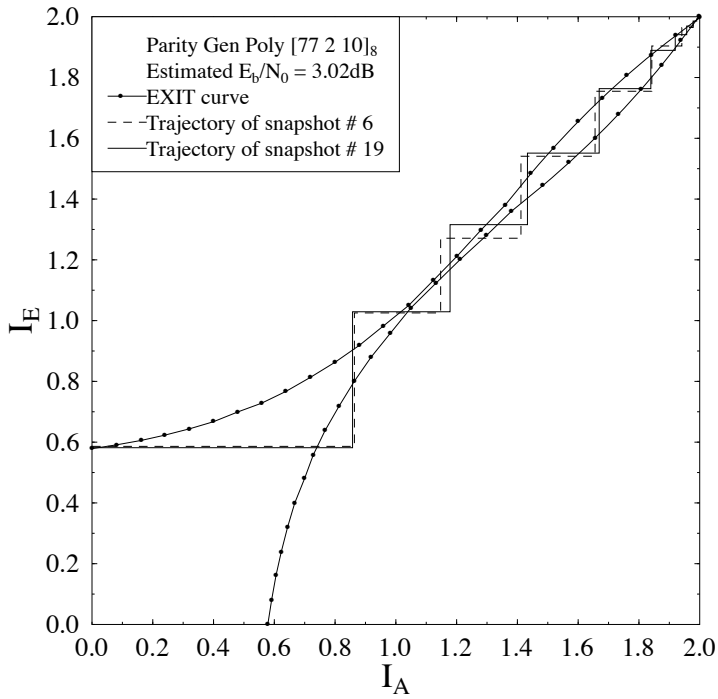

Fig. 5. EXIT chart and two snapshot decoding trajectories for half-rate QPSK-assisted SECTCM using a block length of $10^{4}$ symbols and $\nu=5$ at $E_{b} / N_{0}=3.02 \mathrm{~dB}[92]$.

and IV. Since these are identical components, we only have to compute the EXIT curve of one component and the other is its mirror image with respect to the diagonal line. The stair-case-shaped trajectories correspond to the Monte-Carlo simulation based decoding trajectories, when iterating between the two component decoders of the SECCC scheme. We will show in Section III-C that a self-concatenated decoder can be viewed as a parallel-concatenated decoder having two identical 'hypothetical' component decoders each exchanging extrinsic information with the other, although physically there is only one decoder. The EXIT curves of the hypothetical decoder components are plotted within the same EXIT chart together with their corresponding decoding trajectory for the sake of visualizing the transfer of extrinsic information between the decoders. The major scientific contributions on iterative detection and its convergence analysis are summarised in Tables II and III.

Symbol-based EXIT charts of non-binary serial and parallel concatenated schemes have been studied in [122], [123] and [124], respectively. Near-capacity codes have been designed with the aid of EXIT charts in [108] and [125]. A tutorial introduction to EXIT charts may be found in [126]. The concept of EXIT chart analysis has been extended to three-stage concatenated systems in [105], [110], [114].

\section{ITERATIVELY DECODED BINARY Self-Concatenated Convolutional Codes}

In this section we will design various SECCC and SECCCID schemes. We invoke 2D- and 3D-bit-based EXIT charts, respectively. It will be shown that flexible bit-based SECCC schemes can be designed using the proposed method, which is not possible for the symbol-based SECTCM schemes of [92]. 
TABLE II

MAJOR CONCATENATED SCHEMES AND ITERATIVE DETECTION (1962-2000).
TABLE III

MAJOR CONCATENATED SCHEMES AND ITERATIVE DETECTION (2001-2009).

\begin{tabular}{|c|c|}
\hline Year & Milestone \\
\hline 1962 & Gallager invented LDPC codes [22]. \\
\hline 1966 & Forney [25] proposed a novel concatenated coding scheme. \\
\hline 1974 & $\begin{array}{l}\text { Bahl et al. [38] invented the Maximum A-Posteriori Probability } \\
\text { (MAP) algorithm. }\end{array}$ \\
\hline 1990 & Koch and Baier describe a reduced complexity MAP algorithm [50]. \\
\hline 1993 & Berrou, Glavieux, and Thitimajshima invented the TCs [4]. \\
\hline 1994 & $\begin{array}{l}\text { Erfanian, Pasupathy and Gulak describe the Max-log-MAP algorithm } \\
\text { [55]. }\end{array}$ \\
\hline 1995 & $\begin{array}{l}\text { Robertson et al. [58] proposed the log-MAP algorithm that results in } \\
\text { similar performance to the MAP algorithm but at a significantly lower } \\
\text { complexity. } \\
\text { Divsalar et al. [93] applied turbo principle to multiple PCCCs. } \\
\text { Douillard et al. [94] presented turbo equalisation, where iterative } \\
\text { decoding was invoked for exchanging extrinsic information between a } \\
\text { soft-output symbol detector and an outer channel decoder in order to } \\
\text { overcome the multipath propagation effects in Gaussian and Rayleigh } \\
\text { channels. }\end{array}$ \\
\hline 1996 & $\begin{array}{l}\text { Benedetto et al. [95] extended the turbo principle to serially } \\
\text { concatenated block and convolutional codes. }\end{array}$ \\
\hline 1997 & $\begin{array}{l}\text { Loeliger proposed turbo-like codes using a single trellis for their } \\
\text { decoding [89]. } \\
\text { Benedetto } \text { et al. [96] proposed an iterative detection scheme where } \\
\text { iterations were carried out between the outer convolutional code and an } \\
\text { inner TCM decoder. } \\
\text { Caire } \text { et al. } \text { [97], [98] presented the BICM concept along with its } \\
\text { design rules. } \\
\text { Ritcey and Li [64] introduced Bit-Interleaved Coded Modulation using } \\
\text { Iterative Decoding (BICM-ID). }\end{array}$ \\
\hline 1998 & $\begin{array}{l}\text { Robertson and Wörz introduced turbo trellis-coded modulation (TTCM) } \\
\text { [65]. } \\
\text { Benedetto et al. [84], [88] studied multiple SCCCs combined with } \\
\text { interleavers. } \\
\text { Benedetto et al. proposed self-concatenated trellis coded modulation } \\
\text { (SECTCM) schemes [88]. } \\
\text { ten Brink et al. } \text { [99] introduced a soft demapper between the } \\
\text { multilevel demodulator and the channel decoder in an iteratively detected } \\
\text { coded system. }\end{array}$ \\
\hline 1999 & $\begin{array}{l}\text { Wang et al. }[100] \text { proposed iterative multiuser detection and channel } \\
\text { decoding for coded CDMA systems. } \\
\text { Acikel and Ryan [101] designed high-rate punctured TCs. }\end{array}$ \\
\hline 2000 & $\begin{array}{l}\text { Divsalar et al. }[102],[103] \text { employed unity-rate inner codes for designing } \\
\text { low-complexity iterative schemes for bandwidth/power limited } \\
\text { systems having stringent BER requirements. } \\
\text { ten Brink [90] proposed the employment of EXIT charts for analysing } \\
\text { the convergence behaviour of iteratively detected systems. } \\
\text { several decoding algorithms using message passing ideas. }\end{array}$ \\
\hline
\end{tabular}

We detail the proposed design procedure using 2D-EXIT charts in Section III-A1 and 3D-EXIT charts in Section III-B2. It will be argued that bit-based SECCCs lend themselves to more accurate EXIT-chart-based design than their symbolbased SECTCM counterparts shown in Figure 5, because the bits of a SECTCM symbol are not uncorrelated with each other, although this independence would be a prerequiste for having an accurate match between the EXIT curves and the Monte-Carlo simulation based decoding trajectories. Finally, in Section III-C we derive the union bounds for an SECCC scheme, which constitutes an upper bound on the bit error probability. Our derivation is based on the concept of the socalled uniform interleavers used in [127] for PCCC [4] and SCCC [65], [82], [84] in order to analyse their error floor.

\section{A. Binary SECCC}

SECCCs constitute low-complexity schemes involving only a single encoder and a single decoder. An EXIT chart based analysis of the iterative decoder provides an insight into its decoding convergence behaviour and hence it is helpful for finding the best coding schemes for creating SECCCs. An SECTCM scheme was designed using TCM as constituent

\begin{tabular}{|c|c|}
\hline Year & Milestone \\
\hline 2001 & $\begin{array}{l}\text { Lee [104] studied the effect of precoding on SCCC systems for } \\
\text { transmission over ISI channels. } \\
\text { ten Brink [91], [105] extended the employment of EXIT charts to three- } \\
\text { stage PCCCs. } \\
\text { El Gamal } \text { et al. [106] used SNR measures for studying the convergence } \\
\text { behaviour of iterative decoding. } \\
\text { Ramamurthy and Ryan [107] proposed the serial concatenation of } \\
\text { convolutional differential encoders (accumulate codes), whose } \\
\text { performance is better than those of PCCCs. }\end{array}$ \\
\hline 2002 & $\begin{array}{l}\text { Tüchler et al. [108] simplified the computation of EXIT charts. } \\
\text { Tüchler et al. [109] compared several algorithms predicting the } \\
\text { decoding convergence of iterative decoding schemes. } \\
\text { Tüchler et al. [110] extended the EXIT chart analysis to three-stage } \\
\text { SCCCs. }\end{array}$ \\
\hline 2004 & $\begin{array}{l}\text { Sezgin et al. [111] proposed an iterative detection scheme, where a } \\
\text { block code was used as an outer code and STBC as an inner code. } \\
\text { Tüchler et al. [112] proposed a design procedure for creating } \\
\text { systems exhibiting beneficial decoding convergence depending on the } \\
\text { block length. }\end{array}$ \\
\hline 2005 & $\begin{array}{l}\text { Lifang et al. [113] showed that non-square QAM constellations } \\
\text { can be decomposed into a parity-check block encoder having a } \\
\text { recursive nature and a memoryless modulator. Iterative decoding was } \\
\text { implemented in combination with an outer code for improving the } \\
\text { system performance. } \\
\text { Brännström et al. [114] considered EXIT chart analysis for multiple } \\
\text { concatenated codes using 3-dimensional charts and proposed a way for } \\
\text { finding the optimal activation order. } \\
\text { Luo and Sweeney proposed the employment of cross-entropy as a novel } \\
\text { method of predicting the convergence threshold of a TC, which achieved } \\
\text { without imposing the usual conditions of either having a Gaussian } \\
\text { distribution for the } a \text { priorilextrinsic information or perfect knowledge } \\
\text { of the source information [115]. } \\
\text { Douillard and Berrou [116] showed that double-binary TCs are capable } \\
\text { of achieving a better performance in comparison to classic TCs [4]. }\end{array}$ \\
\hline 2006 & $\begin{array}{l}\text { Chatzigeorgiou et al. proposed a novel technique of finding the transfer } \\
\text { function of a punctured TC designed for optimal performance [117]. }\end{array}$ \\
\hline 2008 & $\begin{array}{l}\text { Carson } \text { et al. proposed a novel optimal bit-to-symbol mapping scheme } \\
\text { for an 8PSK modulated BICM-ID system for transmission over quasi- } \\
\text { static fading channels [118]. } \\
\text { Ng et al. [119] used EXIT charts and union bound analysis to } \\
\text { compare the performance of near-capacity TTCM schemes. } \\
\text { Maunder } \text { et al. [120] designed irregular variable length codes for } \\
\text { the near-capacity operation of joint source and channel coding aided } \\
\text { systems. }\end{array}$ \\
\hline 2009 & $\begin{array}{l}\text { Berrou et al. }[121] \text { proposed a low-complexity decoding algorithm for } \\
\text { improving the performance of TCs in the 'turbo-cliff' region with the } \\
\text { introduction of a rate-1 post-encoder applied in a classic TC scheme } \\
\text { at the cost of imposing } 10 \% \text { increase in complexity. }\end{array}$ \\
\hline
\end{tabular}

codes with the aid of EXIT charts in [92]. The proposed design was symbol-based, therefore it had the inherent problem of exhibiting a mismatch between the EXIT curve and the bit-bybit decoding trajectory as shown in Figure 5. The main reason for the mismatch was that the EXIT charts were generated based on the assumption that the extrinsic information and the systematic information part of each TCM encoded symbol are independent of each other, which had a limited validity, since both the systematic and the parity bits were transmitted together as a single $2^{n+1}$-ary symbol. More explicitly, the coded bits in each TCM symbol are correlated [123], [124], hence they cannot convey the maximum possible mutual information, which results in an entropy- or capacity-loss. Nonetheless, we found that the EXIT charts of the symbolbased SECCC scheme can be beneficially used, since the actual EXIT chart tunnel is always wider than the predicted EXIT chart tunnel [92]. Hence, the analysis was still valid, since it assisted us in finding the SNR value, where the decoder became capable of operating at an infinitesimally low BER. In the following section we describe the binary SECCC philosophy [128], which eliminates the mismatch inherited by 


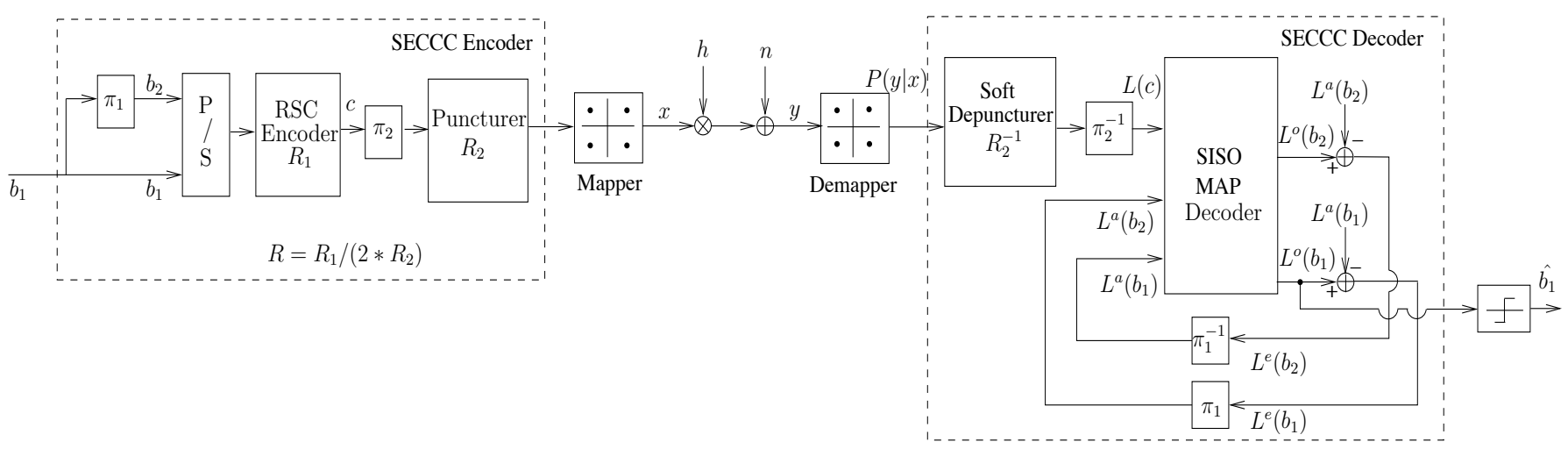

Fig. 6. Binary SECCC scheme [128], which is different from the SECTCM schematic of [92], since the RSC constituent encoder is followed by an interleaver, a puncturer and a modulator. By contrast, in the case of the SECTCM scheme of [92] the TCM constituent encoder generates its output after puncturing the systematic bits. This makes the SECCC scheme more flexible.

the symbol-based TCM design.

The scheme employs binary RSC codes as constituent codes to eliminate the mismatch inherited by the symbol-based TCM design of [92] by proposing a bit-based SECCC design [128] in order to create flexible SECCC schemes capable of efficiently operating over both AWGN and uncorrelated Rayleigh fading channels. Note that when bit-based channel interleaver is employed, each coded bit experiences uncorrelated fading. By contrast, when symbol-based channel interleaver is employed as in the SECTCM scheme, each complex-valued modulated symbol experiences uncorrelated fading but the bits within the modulated symbol experience the same fading. EXIT charts have been used to characterize the convergence behaviour of these schemes. It will be shown that some of the proposed SECCC schemes perform within about $1 \mathrm{~dB}$ from the AWGN and Rayleigh fading channels' capacity. The SECCC schemes considered in Section III-A employ Gray-Mapping (GM) based QPSK modulation. Note that the GM based QPSK can be viewed as two parallel BPSK when communicating over AWGN channel. However, our approach can be extended to higher order modulation schemes including 16QAM and 64QAM, which cannot be viewed as parallel BPSK schemes. The SECCC system model is depicted in Figure 6.

We consider a rate $R=1 / 2$ SECCC scheme as an example, in order to highlight the various system concepts considered in this section. Again, both the AWGN and uncorrelated Rayleigh fading channels are considered. The notation $L($.$) in Figure 6$ represents the LLR of the bit probabilities. The notations $b$ and $c$ in the round brackets (.) in Figure 6 denote the information bits and the coded symbols, respectively. The specific nature of the probabilities and LLRs is represented by the subscripts $a, o$ and $e$, which denote in Figure 6, a priori, a posteriori and extrinsic information, respectively. As shown in Figure 6, the input bit sequence $\left\{b_{1}\right\}$ of the self-concatenated encoder is interleaved for yielding the bit sequence $\left\{b_{2}\right\}$. The resultant bit sequences are parallel-to-serial converted and then fed to the RSC encoder using the generator polynomials $\left(g_{r}=13, g_{1}=\right.$ $\left.15, g_{2}=17\right)_{8}$ expressed in octal format and having a rate of $R_{1}=1 / 3$ and memory $\nu=3$, where $g_{r}$ specifies the feedback polynomial [71]. Hence for every bit input to the SECCC encoder there are six output bits of the RSC encoder. At the output of the encoder there is an interleaver and then a rate $R_{2}=1 / 3$ puncturer, which punctures (obliterates i.e. does not transmit) two bits out of three encoded bits. Hence, the overall code rate, $R$ can be derived based on [129] as:

$$
R=\frac{R_{1}}{2 \times R_{2}}=\frac{1}{2}\left(\frac{1}{3\left(\frac{1}{3}\right)}\right)=\frac{1}{2} .
$$

Therefore, at the output of the puncturer the number of encoded bits reduces from six to two bits, namely to $\left(c_{1} c_{0}\right)$. Puncturing is used in order to increase the achievable bandwidth efficiency $\eta$. It can be observed that different codes can be designed by changing $R_{1}$ and $R_{2}$. These bits are then mapped to a QPSK symbol as $x=\mu\left(c_{1} c_{0}\right)$, where $\mu($. is the Gray-coded mapping function. Hence the bandwidth efficiency is given by $\eta=R \times \log _{2}(4)=1 \mathrm{bit} / \mathrm{s} / \mathrm{Hz}$ assuming a zero Nyquist roll-off-factor. The QPSK symbol $x$ is then transmitted over the communication channel. At the receiver side the received symbol is given by:

$$
y=h x+n,
$$

where $h$ is the channel's non-dispersive fading coefficient and $n$ is the AWGN having a variance of $N_{0} / 2$ per dimension. We assumed that the receiver knows perfectly the channel fading coefficient and the noise variance throughout this paper. This signal is then used by a soft demapper for calculating the conditional PDF of receiving $y$, when a complex-value $x^{m}$ was transmitted, yielding

$$
P\left(y \mid x^{(m)}\right)=\frac{1}{\pi N_{0}} \exp \left(-\frac{\left|y-h x^{(m)}\right|^{2}}{N_{0}}\right),
$$

where $x^{(m)}=\mu\left(c_{1} c_{0}\right)$ is the hypothetically transmitted QPSK symbol for $m \in\{0,1,2,3\}$. Then these PDFs are passed to a soft depuncturer, which converts the PDFs to bit-based LLRs and inserts zero LLRs at the punctured bit positions. These LLRs are then deinterleaved and fed to the SISO MAP decoder. The decoder of Figure 6 is a selfconcatenated decoder. It first calculates the extrinsic LLRs of the information bits, namely $L^{e}\left(b_{1}\right)$ and $L^{e}\left(b_{2}\right)$. Then they are appropriately interleaved to yield the a priori LLRs of the information bits, namely $L^{a}\left(b_{1}\right)$ and $L^{a}\left(b_{2}\right)$, as shown in Figure 6. Self-concatenated decoding proceeds, until a fixed number of iterations is reached. 
1) Decoding Convergence Analysis Using 2-D EXIT Charts: Let us now embark on finding the threshold $E_{b} / N_{0}$ point by calculating the EXIT curve of the identical decoder components and then plotting them together in the EXIT chart, as detailed in [92]. For a code-rate of $R_{1}=1 / 2$ and codememory of $\nu=2$, the generator polynomial $G=(7,5)_{8}$ is used, whereas for $\nu=3$, the generator polynomial $G=(13,15)_{8}$ is employed. For $R_{1}=1 / 3$ and $\nu=3$, $G=(13,15,17)_{8}$ is used, where the first number in the generator polynomial represents the feedback polynomial [71].

The bits are mapped to the QPSK symbols using Gray Mapping (GM). The EXIT charts of the GM-based RSC-coded SECCC systems having rates of $R_{1}=1 / 2$ and $R_{2}=3 / 4$ are plotted in Figure 7. The EXIT curves of the proposed scheme accurately match the decoding trajectories computed from the bit-by-bit simulations, which was not the case for the symbolbased EXIT charts of [92] recorded for the TCM constituent codes.

To elaborate a little further, the EXIT curves and two randomly choosen decoding trajectories were recorded for the specific binary SECCC scheme that was found to operate closest to the Rayleigh channel's capacity in Figure 7. These were recorded by using 10 transmission frames, each consisting of $24 \times 10^{3}$ information bits for calculating the EXIT curve and 10 frames each consisting of $120 \times 10^{3}$ information bits for calculating the decoding trajectories.

For the scheme employing $\nu=3, R_{1}=1 / 2$ and $R_{2}=3 / 4$, the $E_{b} / N_{0}$ distance between the capacity and the threshold $E_{b} / N_{0}$ point where the decoding trajectory always gets through the open tunnel is $1.02 \mathrm{~dB}$ in case of Rayleigh fading channels. For a bandwidth efficiency of $0.67 \mathrm{bit} / \mathrm{s} / \mathrm{Hz}$, the capacity of the $\nu=3, R_{1}=1 / 2$ and $R_{2}=3 / 4$ scheme [2] is $0.54 \mathrm{~dB}$ for the QPSK-based discrete-input Rayleigh fading channels.

\section{B. Binary SECCC-ID Using Soft Decision Demapping}

It was suggested in [131] that a symbol-based scheme always has a lower convergence threshold compared to an equivalent binary scheme. In order to recover the information loss due to employing binary rather than non-binary schemes, we will demonstrate that soft decision feedback is required between the SISO MAP decoder and the soft demapper [130], [132].

Similar to Bit-Interleaved Coded Modulation using the Iterative Decoding (BICM-ID) concept [133], we also employ iterations between the SECCC and the Soft Demapper in our SECCC-ID scheme. However, instead of using $N$ parallel bit interleavers as in BICM-ID, we only have one bit interleaver in our system. Note that the optimized mapping of [134] and the multidimensional mapping of [135] can also be employed for the SECCC-ID scheme.

Two-stage iterative receivers can be analysed with the aid of 2-D EXIT charts, while their three-stage counterparts require 3-D EXIT charts, which were proposed in [110] and further studied in [114], [136], [137]. We will show that 3-D EXIT charts provide a unique insight into the design of near-capacity SECCC-ID codes.

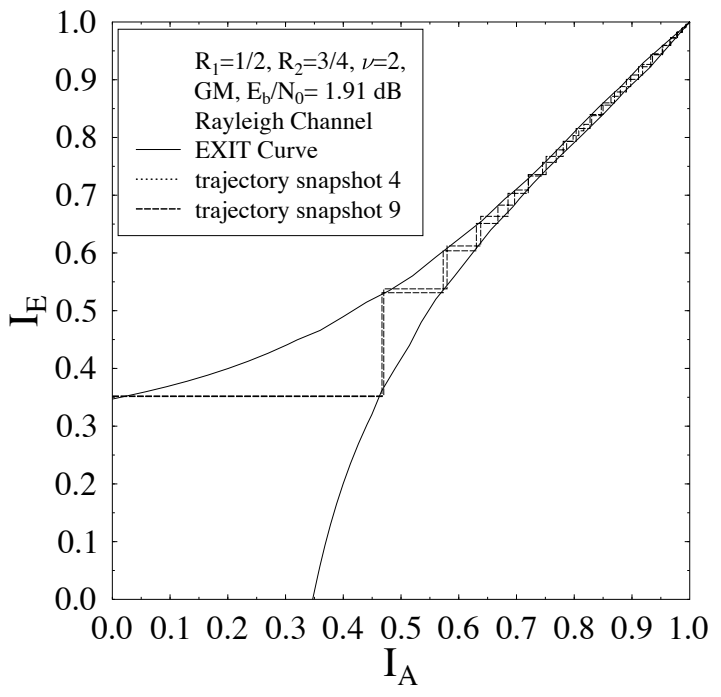

(a) $\nu=2$

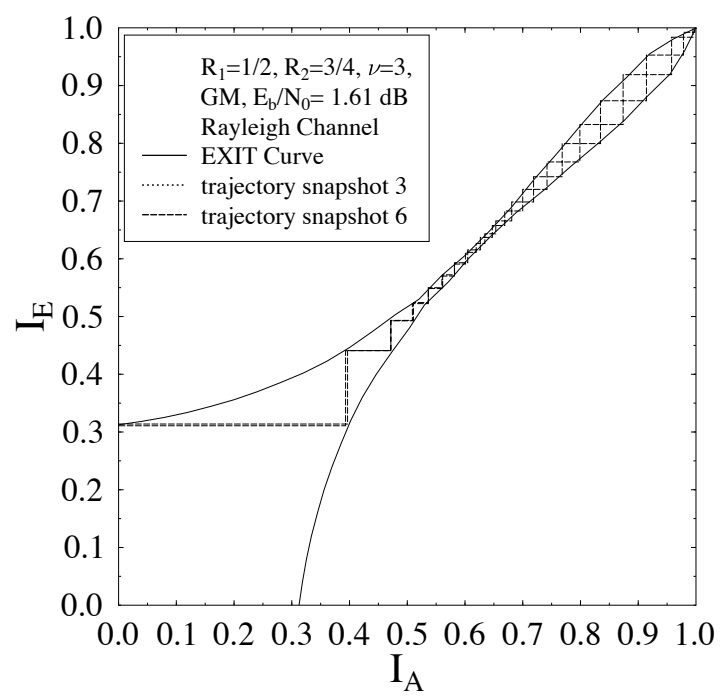

(b) $\nu=3$

Fig. 7. EXIT chart and two 'snap-shot' decoding trajectories for $R_{1}=1 / 2$ and $R_{2}=3 / 4$, QPSK-assisted SECCC, $\eta=0.67 \mathrm{bit} / \mathrm{s} / \mathrm{Hz}$, for transmission over a Rayleigh fading channel.

1) Binary SECCC-ID System Model: The proposed binary SECCC-ID system model employing Set Partitioning (SP) [138] based QPSK modulation is shown in Figure 8. The notations $P($.$) and L($.$) in Figure 8$ denote the logarithmicdomain symbol probabilities and the LLR of the bit probabilities, respectively. The rest of the notations used in Figure 8 have been defined in Section III-A. The binary SECCC scheme depicted in Figure 6 does not have the ability of exchanging soft information with the demapper.

The QPSK symbol $x$ is then transmitted over the communication channel. At the receiver side the received symbol is given by Equation 2. This signal is then used by the demapper for calculating the conditional PDF of receiving $y$, when $x^{m}$ was transmitted, as in Equation 3. These PDFs are then passed through the Symbol to 'Bit Probability Converter' of Figure 8, which first converts the a posteriori symbol probabilities 


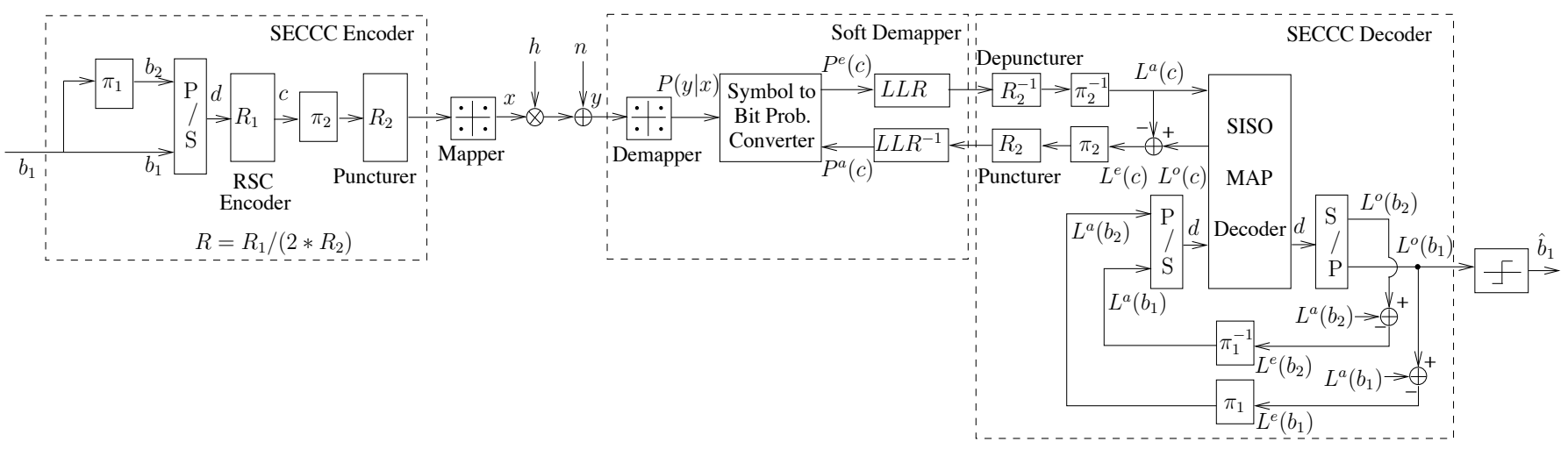

Fig. 8. Binary SECCC-ID system, which is different from the SECCC scheme of Figure 6, since the Soft Demapper in this case exchanges extrinsic information with the SISO MAP decoder [130].

to bit probabilities and then converts those to extrinsic bit probabilities. The extrinsic bit probabilities are then converted to the corresponding bit-based LLRs by the block denoted as $L L R$ in Figure 8, which are then passed through a soft depuncturer inserting zero LLRs at the punctured bit positions. The LLRs are then deinterleaved and fed to the SISO MAP decoder [96].

The self-concatenated decoding procedure is similar to that described in Section III-A. The extrinsic LLRs of the codeword denoted by $L^{e}(c)$ at the output of the SISO decoder are fed back to the Soft Demapper of Figure 8, which are interleaved by $\pi_{2}$ and then punctured according to $R_{2}$. These are then converted to the a priori bit probabilities $P_{b}^{a}(c)$ by the block denoted as $L L R^{-1}$ in Figure 8 , to be fed to the APP demapper, which first converts them to symbol probabilities and then provides the improved extrinsic LLR $L^{e}(c)$ of the codeword at its output, thus completing the outer iteration between the SISO decoder and Soft Demapper. Apart from having inner self-concatenated iterations in the outer SECCC decoder of Figure 8, a fixed number of outer iterations exchange extrinsic information between the decoder and softdemapper to yield the decoded bits $\hat{b}_{1}$.

2) Decoding Convergence Analysis Using 3-D EXIT Charts: Again, EXIT charts constitute powerful tools designed for analysing the convergence behaviour of concatenated codes without time-consuming bit-by-bit simulation of the actual system. Recall that they analyse the mutual information exchange between the input and output of both the inner and outer components of an iterative decoder and find its convergence threshold. The a priori LLRs are modelled either by an AWGN process or by its experimentally determined histogram and then computing the corresponding mutual information between the extrinsic LLRs as well as the corresponding bit-decisions. To make extrinsic LLRs Gaussian distributed EXIT charts require a sufficiently high interleaver length.

EXIT charts [91] are again employed to visualize the input/output characteristics of the constituent SECCC-ID scheme in terms of the average mutual information transfer. The mutual information exchange between the components of an SECCC-ID scheme is portrayed in Figure 9, which shows the SECCC decoder of Figure 8 as two hypothetical component decoders.

As depicted in Figure 9, component 1 and 2 of the
SECCC decoder seen in Figure 8 are associated with four mutual information transfers. Hence two three-dimensional EXIT charts [110], [137] are required for visualising the mutual information transfer between the hypothetical SECCC component decoders (namely for portraying each of the two outputs as a function of two inputs) and the EXIT curve of the combined SECCC decoder and the soft demapper (a two input, single output block).

The $E_{b} / N_{0}$ value, where the two EXIT curves touch each other is termed as the threshold $E_{b} / N_{0}$ point denoted by $\Lambda$, which is the point where the 'turbo-cliff' [4] region starts and beyond which the EXIT tunnel becomes 'just' open, as shown in Figure 7. If uncorrelated extrinsic information is available, then all of the symbol-by-symbol decoding trajectories will reach the $\left(I_{A}, I_{E}\right)=(1,1)$ point [91] for $E_{b} / N_{0}$ values higher than $\Lambda$. The various coding schemes considered in this section are characterised in Table IV. They are identified by the code rate $\left(R_{1}\right)$, puncturing rate $\left(R_{2}\right)$, the overall code rate $(R)$, code memory $\nu$ and bandwidth efficiency $\eta$, expressed in $\mathrm{bit} / \mathrm{s} / \mathrm{Hz}$. Furthermore, $O$ denotes the total number of iterations of SECCC-ID scheme and $I$ denotes the total number of iterations of SECCC scheme. In all the codes considered in Table IV the thresholds are calculated for $O=40$ and $I=40$ for the SECCC and SECCC-ID schemes, respectively. In the case of SECCC the two identical code components iterate 20 times exchanging extrinsic information with each other, while in the case of SECCC-ID the two identical code components iterate 20 times with the demapper. Finally, the channel capacity limit $\omega$ is also expressed in dBs [2], as tabulated in Table IV.

The EXIT charts recorded for the binary SECCC-ID schemes of Table IV are shown in Figures 10, 11 and 12(b). The hypothetical component 2 of the SECCC decoder of Figure 9 receives inputs from and provides outputs for both the soft demapper and the hypothetical component 1 SECCC decoder of Figure 9. Hence we have two EXIT surfaces in Figure 10, the first one corresponding to the component 2 decoder's average mutual information $I_{E_{3}}(C)$ provided for the soft demapper, while the second one corresponding to $I_{E_{3}}(D)$ is supplied for the component 1 SECCC decoder, as shown in Figure 9. The same procedure can be used to calculate the two EXIT surfaces for the average mutual information of the component 1 decoder. One of the EXIT surfaces corresponds to the mutual information $I_{E_{2}}(C)$ provided for the soft demap- 


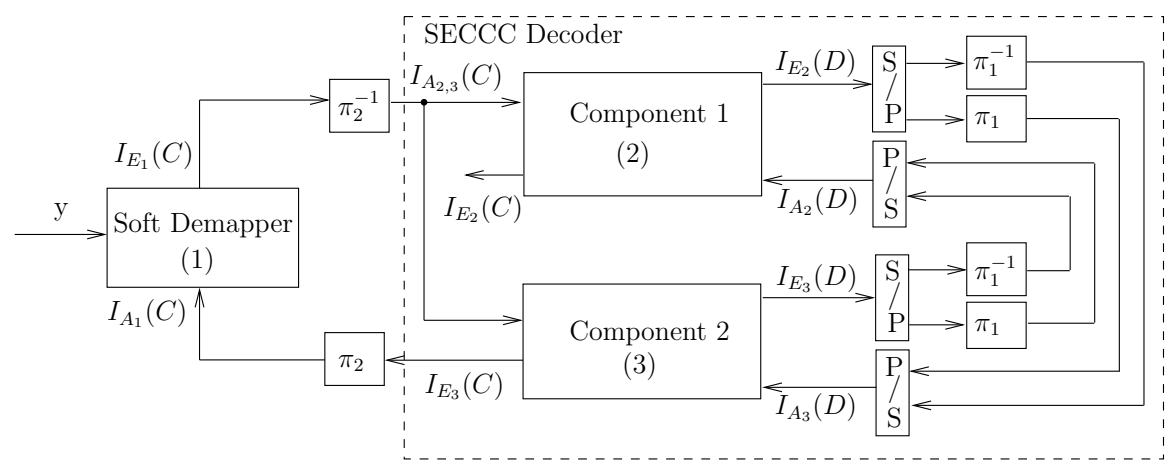

Fig. 9. Mutual information exchange between the three components of an SECCC-ID scheme.

TABLE IV

VARIOUS SECCC AND SECCC-ID SCHEMES AND THEIR THRESHOLDS.

\begin{tabular}{|c|c|c|c|c|c|c|c|}
\hline \multirow{2}{*}{$\begin{array}{l}\text { SECCC/ } \\
\text { SECCC-ID } \\
\text { Schemes }\end{array}$} & \multirow[t]{2}{*}{ Mapping } & \multirow[t]{2}{*}{$\nu$} & \multirow{2}{*}{$\begin{array}{r}\eta \\
\text { (bit/s } \\
/ \mathrm{Hz})\end{array}$} & \multicolumn{2}{|c|}{$\begin{array}{l}\text { AWGN Channel } \\
E_{b} / N_{0}(\mathrm{~dB})\end{array}$} & \multicolumn{2}{|c|}{$\begin{array}{l}\text { Rayleigh Channel } \\
E_{b} / N_{0}(\mathrm{~dB})\end{array}$} \\
\hline & & & & $\Lambda$ & $\omega$ & $\Lambda$ & $\omega$ \\
\hline $\begin{array}{l}\mathrm{R}_{1}=1 / 2, \\
\mathrm{R}_{2}=3 / 4, \\
\mathrm{R}=1 / 3\end{array}$ & $\begin{array}{c}\text { GM } \\
\text { SP } \\
\text { GM } \\
\text { SP }\end{array}$ & 3 & 0.67 & $\begin{array}{r}0.71 \\
\mathbf{0 . 2 5} \\
0.44 \\
0.5\end{array}$ & -0.49 & $\begin{array}{l}1.81 \\
\mathbf{1 . 3 5} \\
1.56 \\
1.55\end{array}$ & 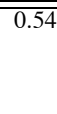 \\
\hline $\begin{array}{l}\mathrm{R}_{1}=1 / 2, \\
\mathrm{R}_{2}=1 / 2, \\
\mathrm{R}=1 / 2\end{array}$ & $\begin{array}{c}\text { GM } \\
\text { SP } \\
\text { GM } \\
\text { SP }\end{array}$ & 2 & 1 & $\begin{array}{r}1.45 \\
\mathbf{1 . 0} \\
1.2 \\
1.25\end{array}$ & 0.19 & $\begin{array}{l}3.4 \\
3.4 \\
\mathbf{3 . 2} \\
3.3\end{array}$ & 1.83 \\
\hline $\begin{array}{l}\mathrm{R}_{1}=1 / 2, \\
\mathrm{R}_{2}=1 / 3, \\
\mathrm{R}=3 / 4\end{array}$ & $\begin{array}{c}\text { GM } \\
\text { SP } \\
\text { GM } \\
\text { SP }\end{array}$ & 2 & 1.5 & $\begin{array}{r}3.44 \\
\mathbf{3 . 2} \\
3.24 \\
3.2\end{array}$ & 1.65 & $\begin{array}{r}8.54 \\
8.4 \\
\mathbf{8 . 0 9} \\
8.1\end{array}$ & 4.98 \\
\hline $\begin{array}{l}\mathrm{R}_{1}=1 / 3, \\
\mathrm{R}_{2}=2 / 3, \\
\mathrm{R}=1 / 4\end{array}$ & $\begin{array}{l}\mathrm{GM} \\
\mathrm{SP}\end{array}$ & 3 & $\overline{0.5}$ & $\begin{array}{l}0.17 \\
0.07\end{array}$ & -0.86 & $\begin{array}{l}0.96 \\
\mathbf{0 . 8 2}\end{array}$ & -0.09 \\
\hline $\begin{array}{l}\mathrm{R}_{1}=1 / 3, \\
\mathrm{R}_{2}=1 / 3, \\
\mathrm{R}=1 / 2\end{array}$ & $\begin{array}{l}\mathrm{GM} \\
\mathrm{SP}\end{array}$ & 3 & 1 & $\begin{array}{l}1.28 \\
1.23\end{array}$ & 0.19 & $\begin{array}{l}3.3 \\
3.4\end{array}$ & 1.83 \\
\hline $\begin{array}{l}\mathrm{R}_{1}=1 / 3, \\
\mathrm{R}_{2}=1 / 4, \\
\mathrm{R}=2 / 3\end{array}$ & $\begin{array}{l}\text { GM } \\
\text { SP }\end{array}$ & 3 & 1.33 & $\begin{array}{l}2.43 \\
\mathbf{2 . 3 7}\end{array}$ & 1.06 & $\begin{array}{r}5.95 \\
5.7\end{array}$ & 3.65 \\
\hline
\end{tabular}

per (not used) in Figure 9. Similarly, the component 1 SECCC decoder has the other EXIT surface characterising its average mutual information $I_{E_{2}}(D)$ forwarded to the hypothetical component 2 SECCC decoder of Figure 9. By contrast, the soft demapper has a single EXIT surface characterising its average mutual information $I_{E_{1}}(C)$ forwarded to component 1 and 2 of the SECCC decoder of Figure 9.

The scheme using $R_{1}=1 / 2, R_{2}=3 / 4, \nu=2$ and employing the SP based Soft Demapper is shown in Figures 10 and 11. Specifically, the EXIT surface marked with triangles in Figure 10 was computed based on the Soft Demapper's output $I_{E_{1}}(C)$ at the given $I_{E_{3}}(D)$ value of the component 2 SECCC Decoder and $I_{A_{1}}(C)$ of the Soft Demapper's abscissa values. By contrast, the steeply rising EXIT surface drawn using dotted lines in Figure 10 was computed based on the component 2 decoder's outputs $I_{E_{3}}(C)$ and $I_{E_{3}}(D)$ at the given $I_{A_{2,3}}(C)$ value. Note that the Soft Demapper characteristic is independent of $I_{E_{3}}(D)$ gleaned from the output of the component 2 decoder, as seen in Figure 9. As we can see from Figure 10, the decoding trajectory is computed at $E_{b} / N_{0}=1.55 \mathrm{~dB}^{1}$. The Monte-Carlo simulation-based symbol-by-symbol decoding trajectory (solid line) relies on the average mutual information of the component 2 SECCC decoder's output, namely on $I_{E_{3}}(C)$, and it evolves within the space under the EXIT surface marked with triangles but above the EXIT surface drawn using dotted lines, which means that it matches the 3-D EXIT curves.

Similarly, the EXIT surface of Figure 11 spanning from the horizontal line $\left[I_{A_{2}}(D)=\{0 \rightarrow 1\}, I_{E_{2}}(D)=0\right.$, $\left.I_{A_{2,3}}(C)=0\right]$ to the horizontal line $\left[I_{A_{2}}(D)=\{0 \rightarrow 1\}\right.$, $\left.I_{E_{2}}(D)=1, I_{A_{2,3}}(C)=1\right]$, represents the first hypothetical SECCC decoder component. Since in case of SECCCs these are identical components, we only have to compute the EXIT surface of a single component and the other is its mirror image [92]. The EXIT surfaces of the two hypothetical decoder components are plotted within the same EXIT chart together with their corresponding decoding trajectory for the sake of visualizing the exchange of extrinsic information between the decoders. The EXIT surfaces of the proposed scheme match exactly the decoding trajectories computed from the bit-by-bit simulations.

The 2-D EXIT curves recorded for a Rayleigh fading channel are shown in Figure 12(a). These exemplify the method of finding thresholds for the Gray mapped SECCC-ID scheme using $\nu=2, R_{1}=1 / 2$ and $R_{2}=3 / 4$. 2-D EXIT curves have been used for the case of Gray mapping, because there is no mutual information exchange gain between the soft demapper and the decoder. Hence, the decoder's convergence threshold can be calculated using 2-D EXIT charts for the case of Gray mapping. The threshold of $E_{b} / N_{0}=1.81 \mathrm{~dB}$ is shown in Figure 12(a) and in Table IV, which is $1.27 \mathrm{~dB}$ away from the Rayleigh fading channel's capacity.

By contrast, to calculate the threshold of a given SP mapping based SECCC-ID scheme, we have to rely on 3-D EXIT charts to analyse the mutual information exchange gain achieved, while iterating between the soft demapper and the decoder. This is shown in Figures 10 and 11. The intersection of the surfaces in Figure 10 represents the points of convergence between the SNR-dependent soft demapper and the SNR-independent SECCC-ID decoder. At these intersection points we have shown a solid line. The corresponding $I_{E_{2}}(D)$

${ }^{1}$ Note that there is a small but still beneficial vertical step in the decoding trajectory (Figs. 10 and 11) after each iteration of the SECCC decoder and the Soft Demapper. This justifies the use of 3-D EXIT charts as compared to 2-D EXIT charts, where this gain cannot be observed. 


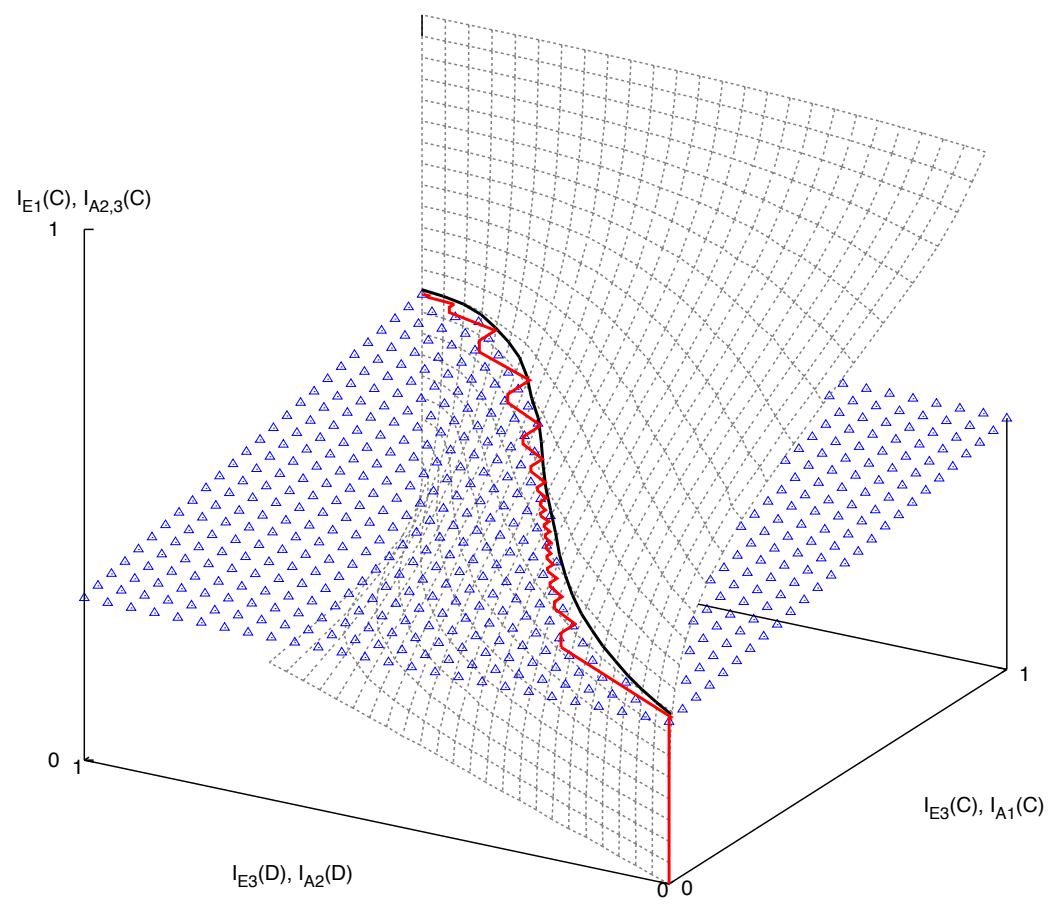

Fig. 10. 3-D EXIT surfaces of SECCC decoder (dotted) and Soft Demapper (triangles) along with a 'snap-shot' decoding trajectory for $R_{1}=1 / 2$ and $R_{2}=3 / 4$, QPSK-assisted SECCC-ID, $\nu=2, \eta=0.67 \mathrm{bit} / \mathrm{s} / \mathrm{Hz}$ at $E_{b} / N_{0}=1.55 \mathrm{~dB}$ using SP mapping, for transmission over an uncorrelated non-dispersive Rayleigh fading channel.

values associated with the curve of intersection between the surfaces in Figure 10 and its mirror image are projected onto the surfaces seen in Figure 11. Figure 11 also shows the Monte-Carlo-simulation based decoding trajectory matching these EXIT curves. These EXIT curves are projected onto $I_{E_{1}}(C)=0$ for yielding Figure 12(b). The 2-D projection seen in Figure 12(b) for the Rayleigh fading channel has a threshold of $1.35 \mathrm{~dB}$. Hence, an overall gain of $0.46 \mathrm{~dB}$ is attained compared to the Gray mapping performance seen in Figure 12(a). The uncorrelated Rayleigh fading channel's capacity is $0.54 \mathrm{~dB}$ for this scheme, hence, it operates $0.81 \mathrm{~dB}$ away from capacity.

The interleaver, $\pi_{1}$ of Figure 8 is used in all of the schemes considered in Table IV, which renders the information bits, more-or-less uncorrelated. This is a necessary requirement for the generation of accurate EXIT charts, because they require the LLRs of the information bits to be Gaussian distributed. The interleaver used after the RSC encoder of Figure 8, namely $\pi_{2}$, randomises the coded bits before the puncturer.

3) Results and Discussions: The EXIT charts discussed in Section III-B2 were used to find the different-rate near capacity SECCC-ID schemes of Table IV designed for $\nu=$ $\{2,3\}$, when communicating over AWGN and uncorrelated non-dispersive Rayleigh fading channels.

The vanishingly-low BER threshold predicted by the EXIT chart analysis detailed in Section III-B2 closely matches with the actual Monte-Carlo-simulation-based threshold observed in the BER curve given by the specific $E_{b} / N_{0}$ value, where there is a sudden drop of the BER after a certain number of decoding iterations, as shown in Figure 13. Hence it becomes possible to attain an infinitesimally low BER beyond the threshold $E_{b} / N_{0}$ value, provided that the block length is sufficiently long and the number of decoding iterations is sufficiently high.

Again, the BER versus $E_{b} / N_{0}$ performance curves of the best performing QPSK-assisted SECCC-ID schemes having $R_{1}=1 / 2$ and $R_{2}=3 / 4$, recorded from our bit-by-bit MonteCarlo simulations are shown in Figure 13. Explicitly, Figure 13 portrays the $E_{b} / N_{0}$ difference between the channel capacity and the system operating at a BER of $10^{-3}$ marked by dotted lines, which was recorded for the SECCC-ID scheme having a code memory of $\nu=2$. The SP mapping scheme operates $0.93 \mathrm{~dB}$ away from capacity, which is $0.35 \mathrm{~dB}$ better compared to the Gray mapping scheme at a BER of $10^{-3}$.

As we can see by studying Table IV and Figure 13, the BER thresholds are accurately predicted by the EXIT charts. Hence, the binary EXIT chart is useful for finding the best SECCCID schemes that are capable of decoding convergence to a vanishingly low BER at the lowest possible $E_{b} / N_{0}$ value. We apply the same method of calculating the BER thresholds for a range of SECCC-ID schemes, as detailed in Table IV. 


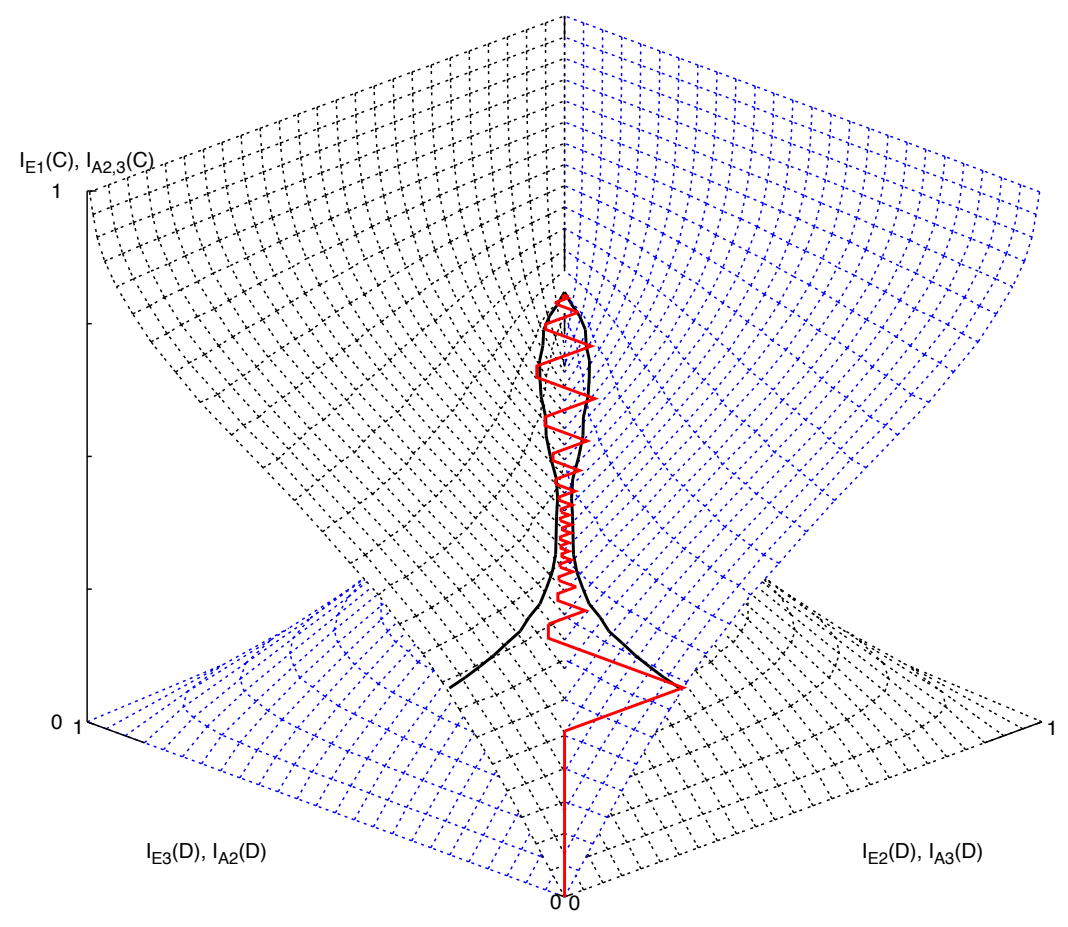

Fig. 11. 3-D EXIT surfaces of the two identical hypothetical SECCC decoder components and a 'snap-shot' decoding trajectory for $R_{1}=1 / 2$ and $R_{2}=3 / 4$, QPSK-assisted SECCC-ID, $\nu=2, \eta=0.67 \mathrm{bit} / \mathrm{s} / \mathrm{Hz}$ at $E_{b} / N_{0}=1.55 \mathrm{~dB}$ using SP mapping for transmission over an uncorrelated non-dispersive Rayleigh fading channel.

\section{Union Bounds of Self-Concatenated Convolutional Codes}

The union bound constitutes a popular code design technique [63], [119], [139]-[143], which may also be used for assisting us in analysing the error floor of turbo-like codes. In this section we derive the union bound of an SECCC scheme for communications over both AWGN and uncorrelated Rayleigh fading channels. As discussed in Section III, the calculation of the union bound involves the computation of the distance spectrum [139] of the code. However, for a high codeword length it may become computationally prohibitive to compute the entire distance spectrum. Hence the Truncated Union Bound (TUB) is considered here, which takes into account the contribution of the lowest non-zero distance spectrum terms [140] rather than only the minimum distance. This technique is useful for studying the corresponding BER floors, regardless of the interleaver lengths [119].

1) System Model for Union Bound Analysis: The schematic of the SECCC encoder employing a $R_{1}=1 / 2$ RSC encoder and a $R_{2}=1 / 2$ puncturer is shown in Fig. 14. As seen from Fig. 14 , the bit sequence $\mathbf{b}_{2}=\left[\begin{array}{llll}b_{2,1} & b_{2,2} & b_{2,3} & \ldots\end{array}\right]$ is simply the interleaved version of the original bit sequence $\mathbf{b}_{1}=\left[\begin{array}{llll}b_{1,1} & b_{1,2} & b_{1,3} & \ldots\end{array}\right]$. After the parallel-to-serial $(\mathrm{P} / \mathrm{S})$ conversion, we can compute the information sequence of the hypothetical upper SECCC component code as $\mathbf{b}^{(1)}=$ $\left[\begin{array}{lllll}b_{1,1} & b_{2,1} & b_{1,2} & b_{2,2} & \ldots\end{array}\right]$. Interestingly, we can view the information sequence of the hypothetical lower SECCC component code $\mathbf{b}^{(2)}$ as the interleaved version of $\mathbf{b}^{(1)}$ using a so-called Odd-Even Separation (OES) based interleaver $\pi_{\text {oes }}$, which was detailed for example in [5]. More explicitly, the OES interleaver consists of two component interleavers, where the odd position of the bit sequence is permuted based on the mapping of $\pi_{o}=\pi$, while the even position of the bit sequence is permuted based on the inverse of the mapping $\pi$, namely on $\pi_{e}=\pi^{-1}$.

We apply a puncturer that removes the interleaved bit sequence $\mathbf{b}_{2}$ as well as all parity bits corresponding to the bit sequence $\mathbf{b}_{1}$ in order to yield the output sequence $\mathbf{c}^{(1)}$, as shown in Fig. 14. The resultant puncturing rate is given by $R_{2}=1 / 2$ and the SECCC output sequence $\mathbf{c}^{(1)}$ consists of only the input bit sequence $\mathbf{b}_{1}$ as well as the parity bit sequence corresponding to $\mathbf{b}_{2}$, as shown in Fig. 14. The SECCC encoder consists of both the rate- $R_{1}$ RSC encoder and the rate$R_{2}$ puncturer. Hence, the coding rate of the SECCC encoder, as shown in Fig. 14, is given by $R=R_{1} /\left(2 R_{2}\right)=1 / 2$.

Based on these observations, we are able to compute the union bound of SECCCs [87], as detailed in Section III-C2.

2) Union Bounds of SECCCs: The so-called Weight Enumerating Function (WEF) is defined as a polynomial, where the weighting coefficient of the $W$-th order term specifies the number of legitimate codewords having a weight of $W$. The WEF of SECCCs may hence be expressed as:

$$
A_{w, \delta}=A_{2 w, \delta^{(1)}}^{(1)} \cdot A_{2 w, \delta^{(2)}}^{(2)} \cdot P_{\pi}^{N, w},
$$




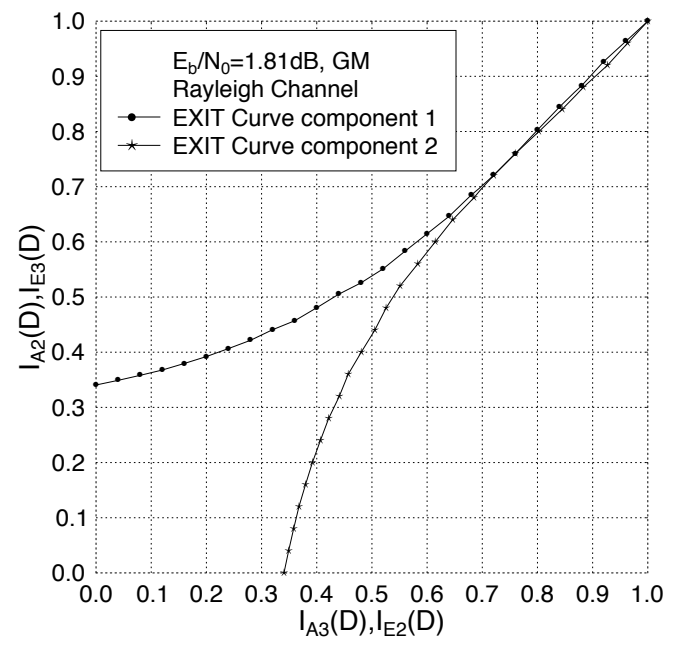

(a) Gray mapping

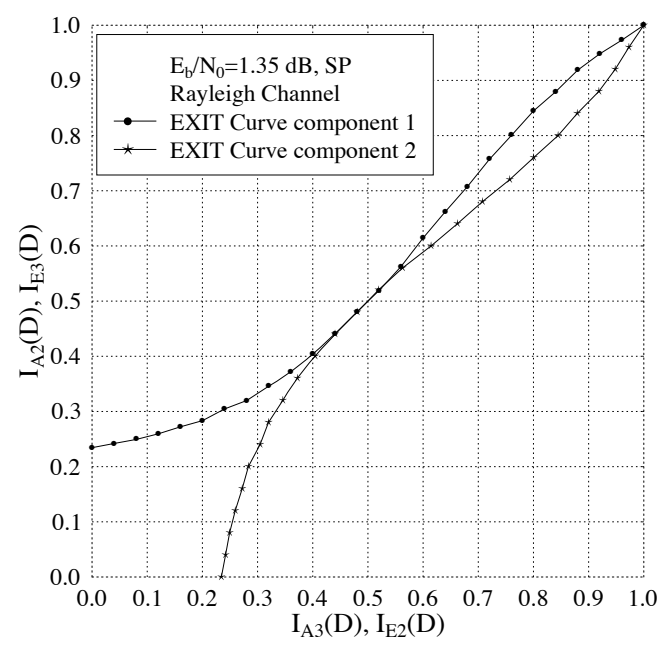

(b) 2D projection of SP mapping

Fig. 12. EXIT curves of the $R_{1}=1 / 2$ and $R_{2}=3 / 4, \nu=2$, SECCC-ID scheme to find the corresponding thresholds, operating over an uncorrelated non-dispersive Rayleigh fading channel.

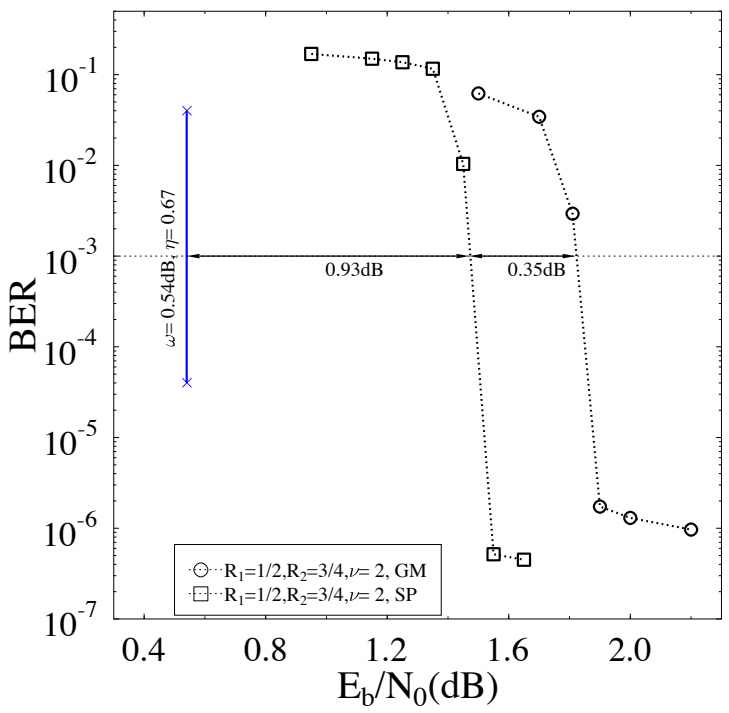

Fig. 13. The BER versus $E_{b} / N_{0}$ performance of Gray and SP mapped QPSK-assisted SECCC-ID schemes, $R_{1}=1 / 2, R_{2}=3 / 4$, and $I=40$ decoding iterations for $\nu=2$, operating over an uncorrelated Rayleigh fading channel.

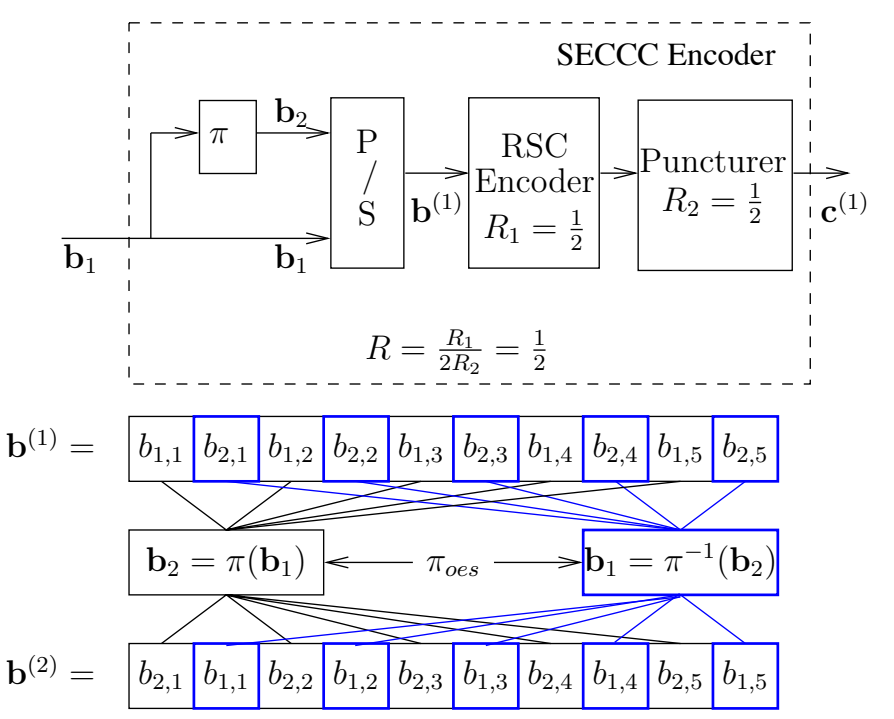

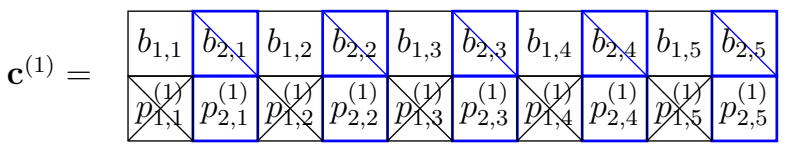

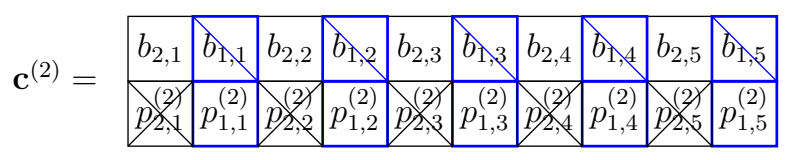

Fig. 14. Schematic of the SECCC encoder. The notations $\mathbf{b}^{(1)}$ and $\mathbf{b}^{(2)}$ denote the information sequences of the hypothetical upper and lower component encoder, respectively, while the puncturer output sequences of the hypothetical upper and lower component encoder are denoted as $\mathbf{c}^{(1)}$ and $\mathbf{c}^{(2)}$, respectively [87].

where $A_{2 w, \delta^{(1)}}^{(1)}$ and $A_{2 w, \delta^{(2)}}^{(2)}$ are the WEFs of the hypothetical upper and lower component codes, respectively. The effective parity weight of an SECCC is given by:

$$
\delta=\delta^{(1)}+\delta^{(2)}
$$

where $\delta^{(1)}$ and $\delta^{(2)}$ are the parity weights of the hypothetical upper and lower component codes, respectively. The above procedure is similar to that devised for the TTCM scheme of [119] employing two TCM constituent codes, where the parity bits of the upper and lower TCM encoded symbols are punctured at the even and odd symbol indices, respectively. As we can see from Fig. 14, the information sequence of the upper component encoder $\mathbf{b}^{(1)}$ consists of the original information sequence $\mathbf{b}_{1}$ and its interleaved version $\mathbf{b}_{2}$. Hence, if the original information sequence $\mathbf{b}_{1}$ has an information weight of $w$, then the information sequence of the upper component encoder $\mathbf{b}^{(1)}$ will have an information weight of $2 w$. The same also applies to the lower component code.

The term $P_{\pi}^{N, w}$ denotes the probability of occurrence for all the associated error events having $w$ information bit errors, when employing a self-concatenated bit-interleaver having a length of $N$ bits. The evaluation of $P_{\pi}^{N, w}$ is based on the novel uniform self-interleaver concept, which may be interpreted as the extension of the uniform bit-interleaver concept proposed in [127]. A uniform self-interleaver of length $N$ bits is a probabilistic device, which maps a given input sequence of 


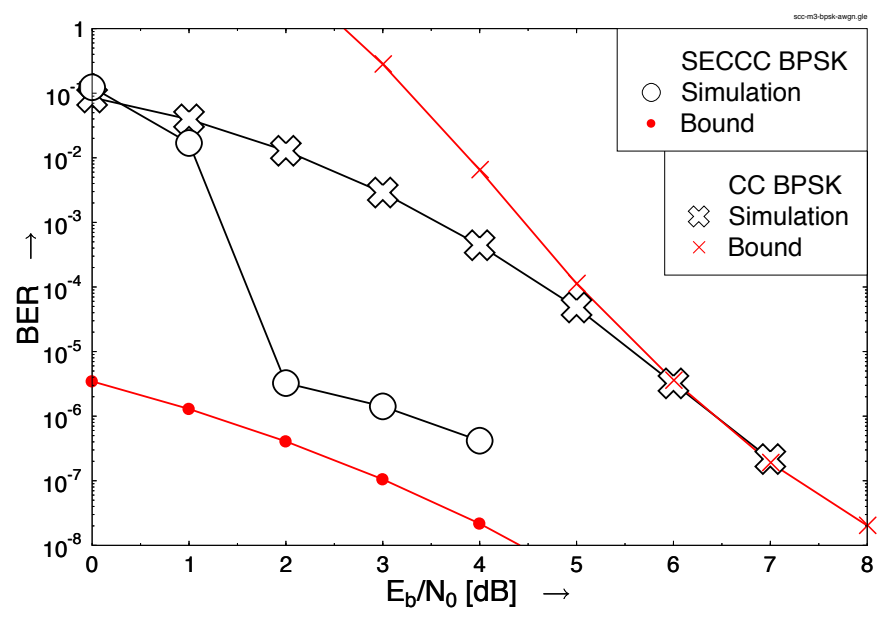

Fig. 15. Simulations and TUBs of BPSK-assisted CC and SECCC, when communicating over AWGN channels. The union bounds are truncated at a maximum Hamming distance of $\Delta_{H \max }=20$. The SECCC employs an interleaver of length 12000 bits and 16 decoding iterations.

length $N$ bits having an information weight of $w$ bits into all possible permutations in the odd and even partitions of an equivalent odd-even-separation based interleaver of length $2 \mathrm{~N}$ having an information weight of $2 w$, with equal probability of:

$$
P_{\pi}^{N, w}=P^{N, w} \cdot P^{N, w},
$$

where $P^{N, w}=1 /\left(\begin{array}{c}N \\ w\end{array}\right)$, which characterizes the traditional $N$ bit uniform interleaver having an information weight of $w$ bits. If there are $w$ bit errors in the information sequence, then there will be $w$ bit errors in the 'odd' sequence $\mathbf{b}_{1}$ as well as another $w$ bit errors in the 'even' sequence $\mathbf{b}_{2}$, since $\mathbf{b}_{2}$ is simply the interleaved version of the $\mathbf{b}_{1}$ sequence.

The WEF $A_{w, \delta}$ of an SECCC having a block length of $N$ encoded symbols and a total of $M$ trellis states can be calculated as follows. We can define the State Input-Redundancy WEF (SIRWEF) for a block of $N$ SECCC-encoded symbols as:

$$
\mathbf{A}(N, S, W, Z)=\sum_{w} \sum_{\delta} A_{N, S, w, \delta} \cdot W^{w} Z^{\delta}
$$

where $A_{N, S, w, \delta}$ is the number of paths in the trellis entering state $S$ at symbol index $N$, which have an information weight of $w$ and a parity weight of $\delta$. The notations $W$ and $Z$ represent dummy variables. For each $n$-bit coded symbol at index $t$, the term $A_{t, S, w, \delta}=\sum_{S^{\prime}, S: u_{t}} A_{t-1, S^{\prime}, w^{\prime}, \delta^{\prime}}$ for $1 \leq t \leq N$ is calculated recursively, where $u_{t}$ represents the specific $k$-bit input symbol that triggers the transition from state $S^{\prime}$ at index $(t-1)$ to state $S$ at index $t$, while the terms $w=w^{\prime}+i\left(S^{\prime}, S\right)$ and $\delta=\delta^{\prime}+\Phi\left(S^{\prime}, S\right)$,

where $w^{\prime}$ and $\delta^{\prime}$ are the information weight and the parity weight, respectively, of the trellis paths entering state $S^{\prime}$ at index $(t-1)$. Furthermore, $i\left(S^{\prime}, S\right) \in\{0,1, \ldots, k\}$ is the information weight of the $k$-bit information symbol $u_{t}$ that triggers the transition from state $S^{\prime}$ to $S$ and $\Phi\left(S^{\prime}, S\right) \in$ $\{0,1, \ldots, n-k\}$ is the parity weight between $\hat{c}_{t}$ and $c_{t}$, where $\hat{c}_{t}$ is the encoded $n$-bit symbol corresponding to the trellis branch in the transition from state $S^{\prime}$ to $S$, while $c_{t}$

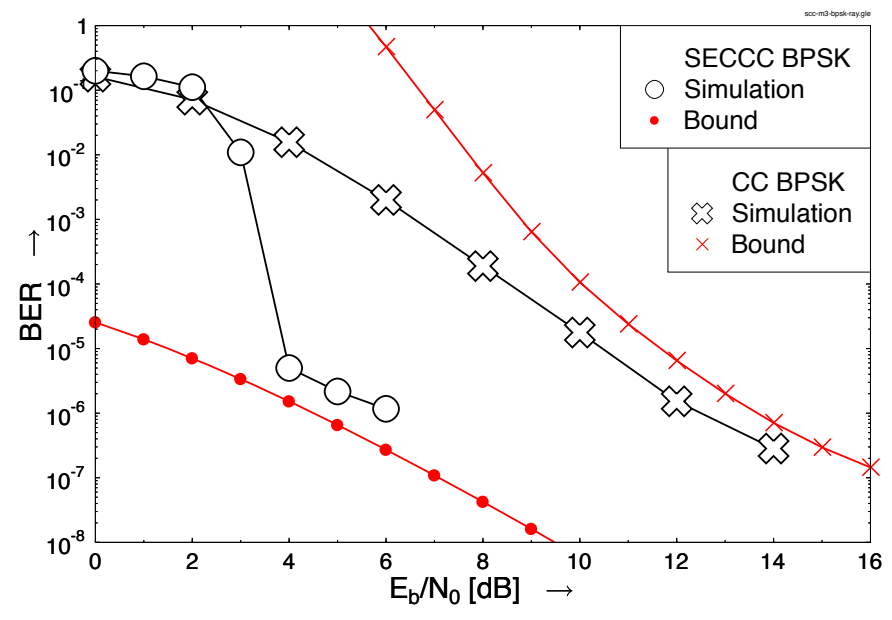

Fig. 16. Simulations and TUBs of BPSK-assisted CC and SECCC, when communicating over uncorrelated Rayleigh fading channels. The union bounds are truncated at a maximum Hamming distance of $\Delta_{H \max }=20$. The SECCC employs an interleaver of length 12000 bits and 16 decoding iterations.

is the actual encoded $n$-bit symbol at index $t$. Again, all the parity bits in $\left\{c_{t}\right\}$ (or $\left\{\hat{c}_{t}\right\}$ ) corresponding to the odd-position information bits are punctured. Note that the parity weight contribution corresponding to a punctured parity bit equals to zero.

Let the encoding process commence from state 0 at index 0 and terminate at any of the $M$ possible states at index $N$. Then the WEF is given by: $A_{w, \delta}=\sum_{S} A_{N, S, w, \delta}$. Note that for linear codes [75] the distance profile of the code is independent of which particular encoded symbol sequence is considered to be the correct one. Hence, for the sake of simplicity, we can assume that the all-zero encoded symbol sequence is transmitted.

The union bound of an SECCC employing BPSK modulation can be shown to be [87]:

$$
P_{b} \leq \sum_{\Delta_{H}} \sum_{w} \frac{A_{2 w, \delta^{(1)}}^{(1)} \cdot A_{2 w, \delta^{(2)}}^{(2)}}{\left(\begin{array}{l}
N \\
w
\end{array}\right) \cdot\left(\begin{array}{l}
N \\
w
\end{array}\right)} \cdot \frac{w \cdot \mathrm{Q}\left(\sqrt{2 \gamma \Delta_{H}}\right)}{k N},
$$

when communicating over AWGN channels and

$$
P_{b} \leq \sum_{\Delta_{H}} \sum_{w} \frac{A_{2 w, \delta^{(1)}}^{(1)} \cdot A_{2 w, \delta^{(2)}}^{(2)}}{\left(\begin{array}{l}
N \\
w
\end{array}\right) \cdot\left(\begin{array}{l}
N \\
w
\end{array}\right)} \cdot \frac{w \cdot(1+\gamma)^{-\Delta_{H}}}{2 k N},
$$

when communicating over uncorrelated Rayleigh fading channels, where $\Delta_{H}=w+\delta^{(1)}+\delta^{(2)}$.

3) Results and Discussions: Let us now compare the BER performance of CCs and SECCCs to their union bounds truncated at a maximum Hamming distance of $\Delta_{H} \max =$ $w_{\max }+\delta_{\max }=20$, where the maximum information and parity weights considered are $w_{\max }=10$ and $\delta_{\max }=10$, respectively. Figures 15 and 16 shows the BERs of our simulations and the corresponding union bounds of the CCs and SECCCs employing BPSK modulation, when communicating over both AWGN and uncorrelated Rayleigh fading channels. Both the CC and SECCC employ an RSC code based on a generator polynomial of $G=\left[\begin{array}{ll}13 & 15\end{array}\right]$ expressed in octal format. 


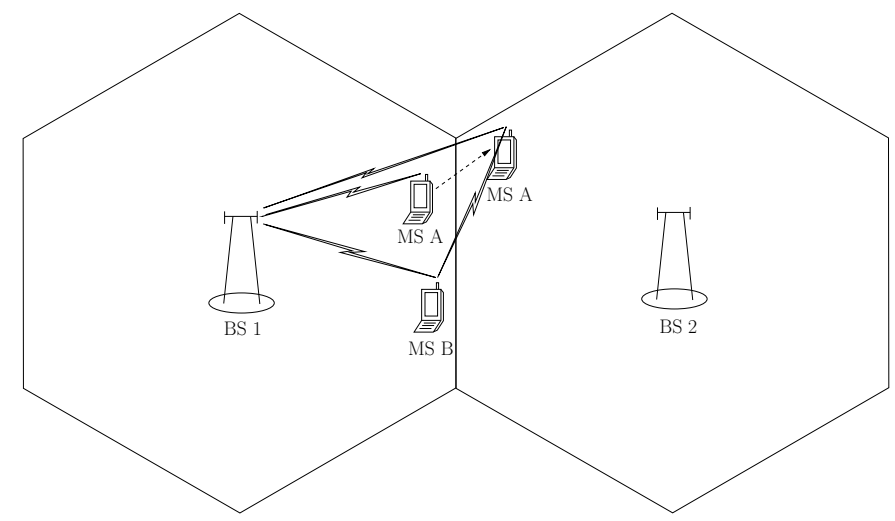

Fig. 17. An example of a cooperative communication scenario.

As shown in Figures 15 and 16, the truncated union bound quantifies the BER floor of SECCCs quite accurately. Hence, we can design SECCCs having various desired BER floors using the proposed TUB.

\section{Distributed Self-Concatenated Coding For COOPERATIVE COMMUNICATIONS}

In this section, we propose a Distributed Binary SelfConcatenated Coding scheme using Iterative Decoding (DSECCC-ID) for cooperative communications [144], which is designed with the aid of binary EXIT charts using the SECCCs of Section III. The benefits of cooperative communications are detailed below.

\section{A. Cooperative Communications}

Traditional direct transmission has its shortfalls, because when the MS roams at the fringe of the cell's coverage region while a conversation is in progress, initiating a handoff might not be possible due to the unavailability of unused channels or the lack of sufficient signal level at the adjacent cell. The call may be dropped in that scenario. Cooperative communication comes to our help in this case. It has the potential of extending the coverage area of a cell by creating an alternative transmission path from the MS to the base station (BS) via the introduction of a relay, as shown in Figure 17. Another advantage of this is the creation of independent paths between the MS and the BS, namely the direct path between the two and the one via the relay.

There are various protocols that may be implemented at the relay channel. These can generally be organised into fixed and adaptive relaying schemes [7]. In fixed relaying schemes the channel resources are shared between the source and the relay in a time-invariant manner. They can be further divided into Amplify-And-Forward (AAF), Decode-And-Forward (DAF), Compress-And-Forward (CAF) and Coded Cooperation [7], [145]. The AAF scheme relies on a relay, which amplifies the received signal and then transmits it to the destination. Although the noise is also amplified along with the signal, we still gain spatial diversity by transmitting the signal over two spatially independent channels [146]. The DAF scheme has a relay which decodes the received signal transmitted by the source, re-encodes it and then forwards it to the destination, which combines all the independently faded signal replicas [146]. In CAF relaying [147], [148] the relay transmits a quantised and compressed version of the received signal in the form of source encoded symbols. At the destination, the source encoded i.e. compressed version of the relay's transmitted signal is decoded by mapping the received bits into a set of values that estimate the source's transmitted message, which are then combined with the message directly received from the source. Finally, in coded cooperation [149] incremental redundancy is introduced by the relay, which is then combined at the destination with the codeword sent by the source, resulting in a codeword benefitting from an increased amount of redundancy. While in some codes the information and redundancy are encoded in such a way that they are inseparable and only perfectly error-free decoding can separate them, some redundancy can be removed from the codeword in the case of punctured concatenated codes.

Major cooperative communications techniques have been outlined in Table V. The basic idea behind cooperative communications can be traced back to the philosophy of the relay channel, which was introduced in 1971 by van der Meulen [150]. Although full-duplex relaying and the associated capacity theorem derived for the discrete memoryless relay channel model have been proposed by Cover and $\mathrm{El}$ Gamal [147], practical cooperative diversity schemes were only proposed much later in [146], [151]-[153]. In [154] Sendonaris et al. generalised the conventional relay model, where there is one source, one relay and one destination, to multiple nodes that transmit their own data as well as serve as relays for each other. The scheme of [154] was referred to as "user cooperation diversity". Sendonaris et al. presented in [151], [155] a simple user-cooperation methodology based on a DAF signalling scheme using CDMA. Dohler et al. [156] introduced the concept of VAAs that emulates Alamouti's STBC for single-antenna-aided cooperating users. Space-time coded cooperative diversity protocols designed for exploiting spatial diversity in a cooperative scenario were proposed in [157]. In practice, each mobile collaborates with either a single or with a few partners for the sake of reliably transmitting both its own information and that of its partners in a concerted action, which emulates a virtual MIMO scheme.

Cooperative communications have been shown to offer significant performance gains in terms of various performance metrics, including improved diversity gains [146], [157], [158] as well as multiplexing gains [159]. Hunter et al. [149] proposed the novel philosophy of coded cooperation schemes, which combine the idea of cooperation with the family of classic channel coding methods. Its extension to the framework of coded cooperation was presented in [8], where the diversity gain of coded cooperation was increased with the aid of ideas borrowed from the area of space-time codes. Additionally, a turbo coded scheme was proposed in [8] in the framework of cooperative communications. The performance benefits of channel codes in a coded cooperation aided scenario were quantified in [160]. Laneman et al. proposed fixed (DAF and $\mathrm{AAF}$ ), selection and incremental relaying protocols and compared them in [146]. 
TABLE V

MAJOR COOPERATIVE COMMUNICATIONS TECHNIQUES (1971-2011).

\begin{tabular}{|c|c|}
\hline Year & Milestone \\
\hline 1971 & $\begin{array}{l}\text { van de Meulen [150] introduced a simple relay channel modeled by three } \\
\text { terminals: a source, a destination and a relay. He studied the problem of } \\
\text { transmission of information as effectively as possible from the source to the } \\
\text { destination assuming that the relay cooperate in the transmission process [161]. }\end{array}$ \\
\hline 1979 & $\begin{array}{l}\text { Cover and El Gamal [147] provided a thorough capacity analysis of the full- } \\
\text { duplex relay channel. }\end{array}$ \\
\hline 1998 & $\begin{array}{l}\text { Sendonaris et al. [154] generalised the relay model to multiple nodes that } \\
\text { transmit their own data as well as serve as relays for each other. }\end{array}$ \\
\hline 2002 & $\begin{array}{l}\text { Hunter et al. [149] introduced coded cooperation to achieve diversity in which } \\
\text { the idea of cooperation was combined with the classic error-control-coding. } \\
\text { Dohler et al. [156] introduced the concept of virtual antenna arrays that } \\
\text { emulates Alamouti's STBC for single-antenna-aided cooperating users. }\end{array}$ \\
\hline 2003 & $\begin{array}{l}\text { Sendonaris et al. [151], [155] presented a simple user-cooperation diversity } \\
\text { based algorithm, where a cooperative CDMA system is implemented. } \\
\text { Laneman et al. [157] developed different cooperative diversity protocols for } \\
\text { exploiting spatial diversity in a cooperation scenario. } \\
\text { Valenti and Zhao [162], [163] proposed a turbo coding scheme in a relay } \\
\text { network. }\end{array}$ \\
\hline 2004 & $\begin{array}{l}\text { Laneman et al. [146] developed cooperative diversity protocols and compared } \\
\text { the performance of DAF, AAF, selection relaying and incremental relaying in } \\
\text { terms of their outage behaviour. } \\
\text { Nabar et al. [164] analysed the spatial diversity performance of various } \\
\text { signalling protocols. } \\
\text { Janani et al. [8] presented two extensions to the coded cooperation framework } \\
\text { [149]: increased the diversity of coded cooperation via ideas borrowed from } \\
\text { space-time codes and applied turbo codes in the proposed relay framework. } \\
\text { Stefanov et al. [160] analysed the performance of channel codes that are } \\
\text { capable of achieving the full diversity provided by user cooperation in the } \\
\text { presence of noisy interuser channels. }\end{array}$ \\
\hline 2005 & $\begin{array}{l}\text { Azarian et al. [159] proposed cooperative signalling protocols that are capable } \\
\text { of striking an attractive diversity-multiplexing tradeoff. } \\
\text { Sneessens et al. [165] proposed a soft decode-and-forward signalling strategy } \\
\text { that can outperform the conventional DAF and AAF. } \\
\text { Hu et al. [166] advocated Slepian-Wolf cooperation that exploits distributed } \\
\text { source coding in wireless cooperative communication. } \\
\text { Yu [167] compared the AAF and DAF signalling schemes in practical scenarios. } \\
\text { Kramer et al. [148] addressed the information-theoretic aspects and considered } \\
\text { DAF and CAF schemes for the wireless relay channels with many relays. }\end{array}$ \\
\hline 2006 & $\begin{array}{l}\text { Hunter et al. }[168],[169] \text { further developed the idea of coded cooperation } \\
\text { [149] by computing BER and FER bounds as well as the outage probability of } \\
\text { coded cooperation. } \\
\mathrm{Li} \text { et al. }[170] \text { employed soft information relaying in a BPSK modulated relay } \\
\text { aided system employing turbo coding. } \\
\text { Hu et al. }[171] \text { proposed Wyner-Ziv cooperation as a generalisation of the } \\
\text { Slepian-Wolf cooperation [166] combined with a compress-and-forward } \\
\text { signalling strategy. } \\
\text { H } \phi \text { st-Madsen [172] derived upper and lower bounds for the capacity of four- } \\
\text { node ad hoc networks having two transmitters and two receivers using } \\
\text { cooperative diversity. }\end{array}$ \\
\hline 2007 & $\begin{array}{l}\text { Bui et al. [173] proposed soft information relaying where the relay's LLR } \\
\text { values are quantised, encoded and superimposed, before being forwarded to } \\
\text { the destination. } \\
\text { Khormuji et al. }[174] \text { improved the performance of the conventional DAF } \\
\text { strategy by employing constellation rearrangement in the source and the relay. } \\
\text { Bao et al. [175] combined the benefits of AAF as well as DAF and proposed } \\
\text { a new signalling strategy referred to as decode-amplify-forward. } \\
\text { Xiao et al. [176] introduced the concept of network coding in cooperative } \\
\text { communications. }\end{array}$ \\
\hline 2008 & $\begin{array}{l}\text { Yue et al. }[177] \text { compared the multiplexed coding and superposition coding in } \\
\text { the coded cooperation system. } \\
\text { Zhang et al. }[178] \text { proposed a distributed space-frequency coded cooperation } \\
\text { scheme for communication over frequency-selective channels. } \\
\text { Wang et al. }[179] \text { introduced the complex field network coding approach that } \\
\text { can mitigate the throughput loss in conventional cooperative signalling schemes } \\
\text { and attain full diversity gain. }\end{array}$ \\
\hline 2009 & $\begin{array}{l}\text { Hanzo et al. [6] presented low-complexity cooperative MIMO codes and } \\
\text { distributed turbo codes designed for two users cooperating for the sake of } \\
\text { improving their attainable BER performance. } \\
\text { Liu et al. }[7] \text { authored a book on cooperative communications and networking. }\end{array}$ \\
\hline 2010 & $\begin{array}{l}\text { Badia et al. [180] analysed coded cooperation and cooperation on medium } \\
\text { access control (MAC) and networking layers. They argue that for crowded } \\
\text { networks coded cooperation suffers due to increase in interference from } \\
\text { neighboring nodes. Similarly, for sparse networks opportunistic routing may } \\
\text { be difficult to achieve. They showed that by combining cooperative routing } \\
\text { with coded cooperation overall system performance can be improved. } \\
\text { Tourki et al. [181] presented a cooperative space-time transmission scheme } \\
\text { where relays cooperate only if the source-relay channel is of an acceptable } \\
\text { quality alongwith suitable power allocation strategies to improve performance. }\end{array}$ \\
\hline 2011 & $\begin{array}{l}\text { Rossetto and Zorzi [182] shed light on the role of coding schemes such as } \\
\text { sphere packing etc. for MIMO aided network coding. They further point } \\
\text { towards several unaddressed issues arising from cross-layer design such as, } \\
\text { building a supportive MAC for physical layer, improving NC encoding phase, } \\
\text { symbol synchronization and modulation schemes to deal with colliding } \\
\text { signals, while keeping the complexity low. }\end{array}$ \\
\hline
\end{tabular}

\section{B. Distributed Coding Techniques}

Distributed coding [183] constitutes another attractive cooperative diversity technique, where joint signal design and coding are invoked at the source and relay nodes. Distributed turbo codes [8], [163] have also been proposed for cooperative communications, although typically under the simplifying assumption of having a perfect communication link between the source and the relay nodes. These are half-duplex relay-aided systems, where the source transmits to both the relay and destination during the first transmission period and after decoding the information from the source the relay re-encodes it and sends it to the destination in the second transmission period. Hence half-duplex systems do not suffer from multiple-access interference, which results in a simplified receiver structure at the cost of halving the spectral efficiency. As a more realistic design alternative, a turbo coded cooperation aided system having an imperfect source-relay (SR) communication link has been proposed in [184], [185]. In [184] the source node continues its transmission of the rest of the codeword in the second transmission period with the aim of achieving an improved bandwidth efficiency. Still referring to [184], the signals arriving from the source and relay are superimposed at the destination, where a Maximum A Posteriori Probability (MAP) detector and a turbo decoder exchange extrinsic information, which were shown to be capable of operating near the capacity of ergodic flat fading channels. The scheme proposed in [185] considers a more complex irregular Low-Density Parity-Check (LDPC) coded near-capacity system designed using EXIT charts and a design-procedure similar to that of [184]. It is demonstrated in [6], [186] that in the presence of Rayleigh fading, DAF cooperation-assisted systems are expected to outperform their non-cooperative counterparts. However, an error floor is observed in [6], which may be mitigated by using soft-relaying [165], [170].

\section{DSECCC-ID System Overview}

The schematic of a two-hop half-duplex relay-aided system is shown in Fig. 18, where the source node $(s)$ transmits a frame of coded symbols $\mathbf{x}_{s}$ to both the relay node $(r)$ and the destination node $(d)$ during the first transmission period $T_{1}$, while the relay node first decodes the information, then reencodes it and finally transmits a frame of coded symbols $\mathbf{x}_{r}$ to the destination node during the second transmission period $T_{2}$. In the Time-Division Multiple Access (TDMA) protocol used, the source transmits to both the relay and destination during $T_{1}$, while in $T_{2}$, only the relay transmits to the destination. The communication links seen in Fig. 18 are subject to both freespace path loss as well as to short-term uncorrelated Rayleigh fading.

In our half-duplex DAF system design study of Fig. 19 the source-destination (SD) link employs a simple SECCC code, while the relay node employs simple RSC encoder instead of SECCC encoder. Therefore, the iterative decoding at the destination exchanges information between the SECCC MAP decoder and an RSC MAP decoder. The source employs a SECCC encoder, which reuses the same component instead of having two separate constituent codes. First SECCC iterative decoding is employed at the relay, which then re-encodes the decoded symbols by a low-complexity RSC encoder. The relay frame is shorter than the source frame because of the puncturing of the systematic bits. Hence, the overall 


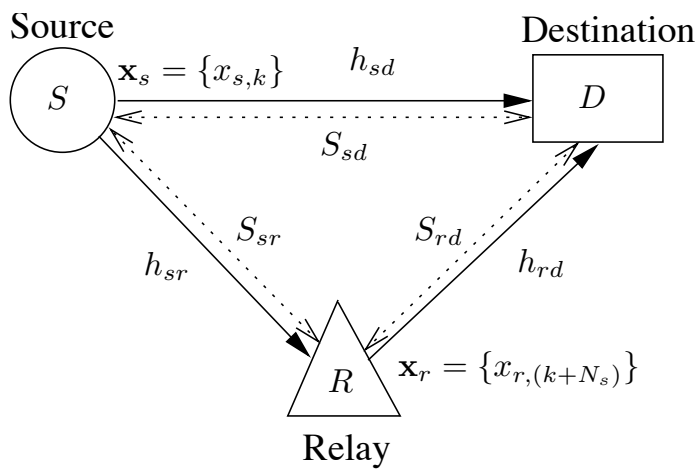

Fig. 18. Schematic of a two-hop relay-aided system, where $S_{a b}$ is the geographical distance between node $a$ and node $b$.

throughput is higher. The motivation for using the proposed 3 -stage decoder architecture is to effectively reduce the errorfloor documented in Figs. 15.6-15.10 of [6] for the conventional two-stage architecture of Fig. 15.3 in [6]. The proposed scheme is designed by a systematic and widely applicable procedure using EXIT charts. The SR link is imperfect, yet this simplified scheme is capable of approaching capacity. We derive the theoretical lower and upper bounds on the Continuous-input Continuous-output Memoryless Channels's (CCMC) capacity as well as of the DCMC [147], [187]-[189] capacity (constrained information rate) for independent and uniformly distributed (i.u.d.) sources.

Let $S_{a b}$ denote the geometrical distance between nodes $a$ and $b$. The path loss between these nodes can be modelled by [153], [162] as $P(a b)=K / S_{a b}^{\alpha}$, where $K$ is a constant that depends on the environment and $\alpha$ is the path loss exponent. For a free-space path loss model we have $\alpha=2$. The relationship between the energy $E_{s r}$ received at the relay node and that of the destination node $E_{s d}$ can be expressed as: $E_{s r}=\frac{P(s r)}{P(s d)} E_{s d}=G_{s r} E_{s d}$, where $G_{s r}$ is the power-gain (or geometrical gain) [153] experienced by the SR link with respect to the SD link as a benefit of its reduced distance and path loss, which can be computed as:

$$
G_{s r}=\left(\frac{S_{s d}}{S_{s r}}\right)^{2}
$$

Similarly, the power-gain for the relay-destination (RD) link with respect to the SD link is given by $G_{r d}=\left(\frac{S_{s d}}{S_{r d}}\right)^{2}$.

1) DSECCC-ID Encoder: In our DSECCC-ID scheme of Fig. 19, we consider the QPSK-assisted SECCC encoder of Fig. 6 in Section III-A at the source and a QPSK-assisted RSC encoder at the relay. As seen in Fig. 19, the relay detects the signals received from the source node using a SECCC scheme during the first transmission period. The notation $\pi_{r}$ in Fig. 19 denotes the random bit interleaver used at the relay to interleave the decoded bits before the RSC encoder. The encoders employed at both the source and relay transceiver nodes may be viewed as a three-component parallel-concatenated SECCC encoder ${ }^{2}$, which is depicted in

\footnotetext{
${ }^{2} \mathrm{An}$ SECCC encoder can be viewed as a two-component parallelconcatenated encoder [87]
}

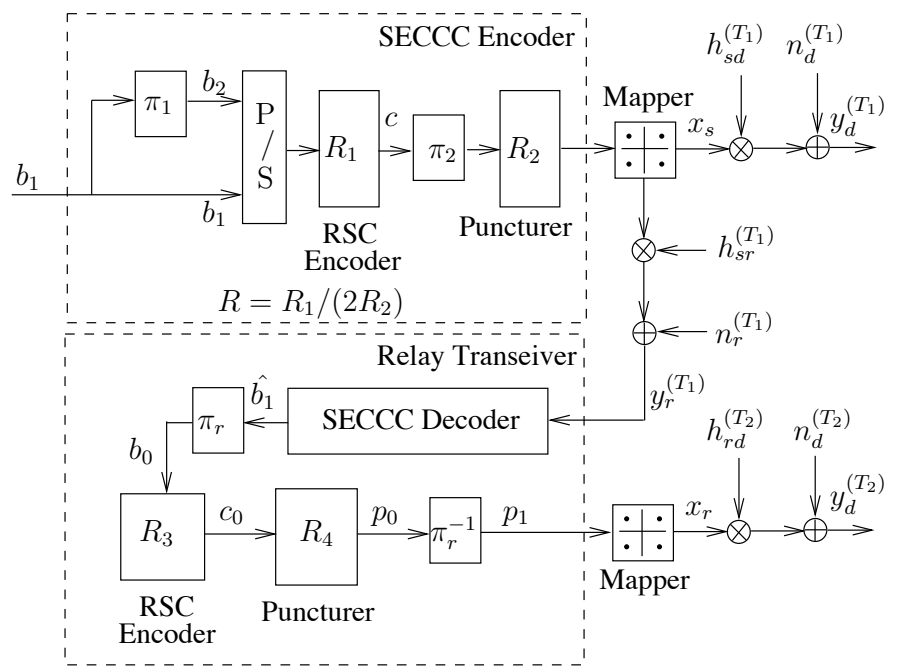

Fig. 19. The schematic of the three-component arrangement using the self-concatenated encoder of Fig. 6. This figure applies to the DSECCC-ID scheme [144], when the relay decodes the received symbols using the SECCC decoder of Fig. 6 and then forwards the decoded symbols to the destination in the second phase.

Fig. 19.

The notation $x_{r}$ used in Fig. 19 denotes the 2-bit QPSK symbol at the relay node. The puncturer denoted as $R_{4}$ in Fig. 19 is used to improve the overall throughput of the scheme by minimizing the number of redundant transmitted bits. We found that a good performance can be achieved by transmitting only the parity bits generated at the output of the RSC encoder at the relay node, while puncturing all the original information bits.

At the source node we consider the rate $R=1 / 3$ SECCC scheme of Fig. 6 in Section III-A operating close to the uncorrelated Rayleigh fading channel's capacity and employing QPSK modulation. As shown in Fig. 19 and discussed in Section III-A, the input bit sequence $\left\{b_{1}\right\}$ of the self-concatenated encoder is interleaved for yielding the bit sequence $\left\{b_{2}\right\}$. The resultant bit sequences are parallelto-serial converted and then fed to the RSC encoder having a rate of $R_{1}=1 / 2$. This stream is then passed through an interleaver and then a rate $R_{2}=3 / 4$ puncturer, as seen in Fig. 19. Hence, the overall code rate evaluated from Equation 1 becomes $R=1 / 3$. These bits are then mapped to a QPSK symbol as $x=\mu\left(c_{1} c_{0}\right)$, where $\mu($.$) is the bit-to-symbol$ mapping function. Hence the bandwidth efficiency is given by $\eta=R \times \log _{2}(4)=0.67$ bits/symbol (bps), assuming a zero Nyquist roll-off-factor. The QPSK symbol $x_{s}$ is then transmitted over the channel. The overall throughput of this two-hop cooperative scheme can be formulated as: $\eta=\frac{N_{i}}{N_{s}+N_{r}}[\mathrm{bps}]$, where $N_{i}$ is the number of information bits transmitted within a duration of $\left(N_{s}+N_{r}\right)$ symbol periods. Again, $N_{s}$ is the number of modulated symbols per frame transmitted from the source node and $N_{r}$ is the number of modulated symbols per frame arriving from the relay node. For our case we have $N_{i}$ $=120,000$ bits. Therefore, we transmit a total of $N_{s}=180,000$ symbols. Note that the number of symbols per transmission burst at the relay node is given by $N_{r}=60,000$ due to the 


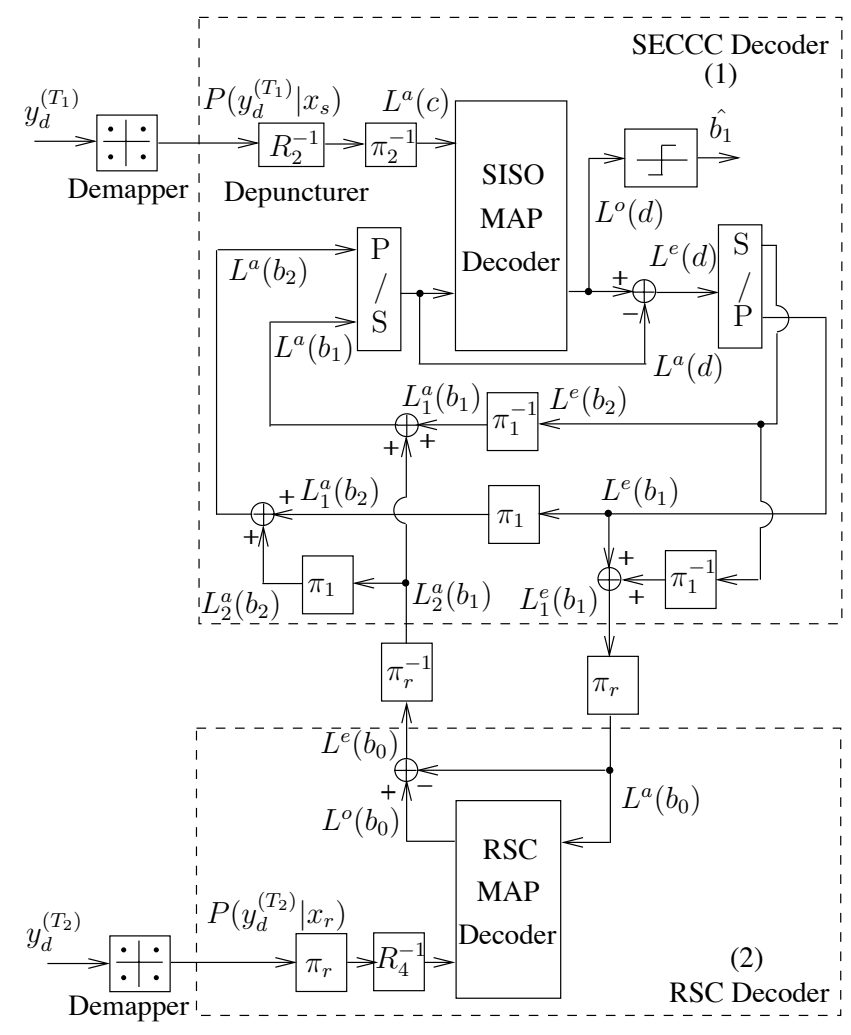

Fig. 20. The schematic of the DSECCC-ID decoder [144]. Note that the SECCC decoder is a modified version of the decoder of Fig. 6 in order to make it capable of exchanging extrinsic information with the RSC decoder. The input of the SECCC decoder is generated by the QPSK demapper for the SD link, while the input of the RSC decoder is output by the QPSK demapper of the RD link.

employment of 2 bps QPSK modulation and a rate $R_{4}=1 / 2$ puncturer that removes all systematic bits from the output of the RSC encoder of rate $R_{3}=1 / 2$. Hence, the overall effective throughput of the DSECCC-ID scheme of Fig. 19 is given by $\eta=\left(N_{i}\right) /\left(N_{s}+N_{r}\right)=0.5$ bps. The Signal to Noise Ratio (SNR) per bit is given by $E_{b} / N_{0}=\mathrm{SNR} / \eta$. Hence, the DSECCC-ID scheme suffers from a penalty of $1.25 \mathrm{~dB}$ in terms of $E_{b} / N_{0}$, when compared to the conventional SECCC scheme having a somewhat higher throughput of $0.67 \mathrm{bit} / \mathrm{s} / \mathrm{Hz}$.

2) DSECCC-ID Decoder: The decoder structure of the DSECCC-ID scheme is illustrated in Fig. 20.

For the SECCC decoder of Fig. 6 and detailed in Section III, which was denoted as component (1) in Fig. 20, the received signal arrives at the soft demapper. This signal is then used by the demapper for calculating the conditional PDF of receiving $\mathbf{y}_{d}^{\left(T_{1}\right)}$, when $\mathbf{x}_{s}^{m}$ was transmitted, where $\mathbf{x}_{s}^{m}=\mu\left(c_{1} c_{0}\right)$ is the hypothetically transmitted QPSK symbol for $m \in\{0,1,2,3\}$, while the received signal that arrives at the soft demapper of the RSC decoder, which is denoted as component (2) in Fig. 20 is used for calculating the conditional PDF of receiving $\mathbf{y}_{d}^{\left(T_{2}\right)}$, when $\mathbf{x}_{r}^{m}$ was transmitted.

The bit probabilities are then passed through a soft depuncturer, which converts them to the corresponding bit-based LLRs and subsequently inserts zero LLRs at the punctured bit positions. The LLRs are then deinterleaved and fed to the Soft-Input Soft-Output (SISO) RSC MAP decoder [96] of Fig. 20.
There are two inputs to the RSC MAP decoder block, which is denoted as component (2) in Fig. 20. The first is the extrinsic information of bit $b_{1}$ provided by the SECCC decoder, which is denoted as component (1). As seen in Fig. 20, this is obtained from the addition of $L^{e}\left(b_{1}\right)$ and the deinterleaved version of $L^{e}\left(b_{2}\right)$. The resultant $L_{1}^{e}\left(b_{1}\right)$ stream is interleaved by $\pi_{r}$ to generate $L^{a}\left(b_{0}\right)$. The second input of the RSC MAP decoder component (2) is the interleaved and depunctured version of the soft information provided by the QPSK demapper denoted as $P\left(y_{d}^{T_{2}} \mid x_{r}\right)$ in Fig. 20. The RSC decoder of the relay seen in Fig. 20 then provides the improved extrinsic LLR of the data bit $b_{0}$, namely $L^{e}\left(b_{0}\right)$ as its output, which is deinterleaved by $\pi_{r}^{-1}$ to yield $L_{2}^{a}\left(b_{1}\right)$. The LLR $L_{2}^{a}\left(b_{1}\right)$ can be further interleaved using $\pi_{1}$ to generate $L_{2}^{a}\left(b_{2}\right)$. These a priori LLRs output by the RSC can be added to the SECCC decoder's a priori LLRs of $b_{1}$ and $b_{2}$, thus completing the iteration between the RSC and SECCC decoders of Fig. 20.

It has been shown in [87] that an SECCC scheme may be viewed as two parallel-concatenated codes separated by an odd-even turbo interleaver. Hence the SECCC Decoder (1) of Fig. 20 employed at the destination may be viewed as a two-component PCCC decoder, which exchanges extrinsic information with another parallel-concatenated RSC Decoder (2) as shown in Fig. 20. Therefore, our proposed scheme can be viewed as a three-component parallel-concatenated scheme.

\section{EXIT Chart Analysis}

Our three-step design procedure using EXIT charts developed for the proposed distributed coded system is as follows:

Step 1: Our code design procedure commences by calculating the decoding convergence threshold of the SECCC scheme at the output of the SR link using EXIT charts. Recall that the near-capacity QPSK-assisted SECCC scheme of Fig. 6 in Section III employs $R_{1}=1 / 2, R_{2}=3 / 4$ and has a throughput of $\eta=0.67 \mathrm{bit} / \mathrm{s} / \mathrm{Hz}$. As seen in Fig. 21, we compare the SECCC scheme using $\nu=2$ and $\nu=3$ at a receive SNR of about $-0.15 \mathrm{~dB}$. For the $\nu=3-\mathrm{SECCC}$ code a receive SNR of about $-0.15 \mathrm{~dB}$ is needed in order to attain a decoding convergence to the $(1,1)$ point of the EXIT chart, since at a receive SNR of $-0.2 \mathrm{~dB}$ the EXITtunnel remains closed. By contrast, the EXIT tunnel for the $\nu=2$-SECCC code, employing the generator polynomial $\left(g_{r}=7, g_{1}=5\right)_{8}$, remains closed at $-0.15 \mathrm{~dB}$. This can also be confirmed from Table IV, where the Gray mapped $\nu=3$ SECCC scheme performs $0.25 \mathrm{~dB}$ better than the $\nu=2$ scheme. Recall from Table IV that the successfuldecoding convergence threshold $\omega$ of a $\nu=2$ code is $1.81 \mathrm{~dB}$ in terms of $E_{b} / N_{0}$, whereas the $\nu=3$ code requires an $E_{b} / N_{0}$ value of $1.56 \mathrm{~dB}$. Consequently, we opted for $\nu=3$, employing the octally represented generator polynomial of $\left(g_{r}=13, g_{1}=15\right)_{8}$, as it requires a marginally reduced transmission power. We hasten to add that this may be deemed an unfavourable tradeoff, since it implies doubling the number of trellis states, i.e. the complexity.

Fig. 21 also corresponds to the performance of the SECCC scheme of the SR link. The receive SNR can be computed as: $\mathrm{SNR}_{r}=\mathrm{SNR}_{e}+10 \log _{10}\left(G_{s r}\right)$ [dB]. When there is 


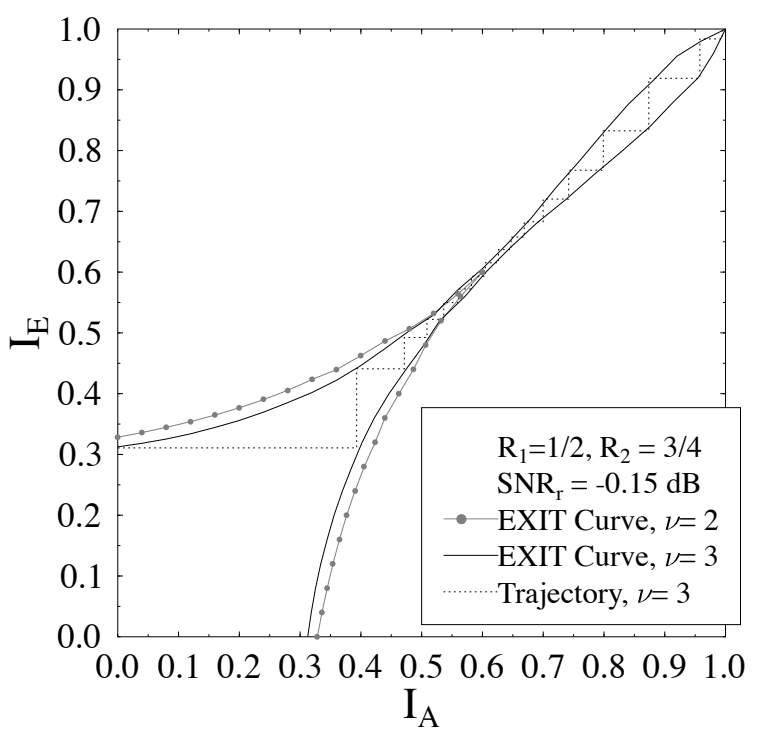

Fig. 21. EXIT curves for $\nu=2$ and $\nu=3, R_{1}=1 / 2$ and $R_{2}=3 / 4$, QPSKassisted SECCC using Gray mapping, $\eta=0.67 \mathrm{bit} / \mathrm{s} / \mathrm{Hz}$ at $\mathrm{SNR}_{r}=-0.15 \mathrm{~dB}$ for transmission over an uncorrelated Rayleigh channel. A decoding trajectory for $\nu=3$ is also depicted. The 2-D EXIT chart is similar to that calculated for the SECCC scheme in Fig. 7(b) at an equivalent $E_{b} / N_{0}$ value of $1.61 \mathrm{~dB}$

no path-loss, the receive SNR equals the equivalent $S N R^{3}$, denoted by $\mathrm{SNR}_{e}$ and $G_{s r}$ was defined in Equation 10. Hence, a receive SNR of $-0.15 \mathrm{~dB}$ can be achieved by various combinations of $\mathrm{SNR}_{e}$ and $G_{s r}$. For the $\nu=3$ SECCC code the successful decoding convergence threshold ${ }^{4}$ is at $-0.2 \mathrm{~dB}$, when employing $I=40$ self-concatenated iterations, which is $1.05 \mathrm{~dB}$ away from the Rayleigh fading SR link's capacity calculated as $-1.20 \mathrm{~dB}$ at 0.67 bps from [2]. The corresponding capacity curve will be discussed later in detail in the context of Fig. 24. This scheme acquires an open EXIT tunnel ${ }^{5}$ at $\mathrm{SNR}_{r}=-0.15 \mathrm{~dB}$, when communicating over an uncorrelated Rayleigh fading channel.

In our analysis the relay node of the DSECCC-ID is assumed to be placed half-way between the source and relay nodes, i.e. we have $G_{s r}=G_{r d}=4$, hence the minimum required equivalent $\mathrm{SNR}$ at the source node is $\mathrm{SNR}_{e}=$ $-0.15-6.02=-6.17 \mathrm{~dB}$.

Step 2: In this section the decoding convergence of the three-component DSECCC-ID decoder used at the destination node is analysed. The EXIT curves of the SECCC decoder at the SD link employing $I_{s d}=2$ self-concatenated iterations

\footnotetext{
${ }^{3}$ To simply our analysis the term "equivalent SNR" is introduced, which is the ratio of the signal power at the transmitter (source/relay node) with respect to the noise level at the receiver (relay/destination node).

${ }^{4}$ The decoding threshold is the SNR value beyond which the EXIT tunnel becomes 'just' open, although this does not necessarily imply that the $\left(I_{A}\right.$, $\left.I_{E}\right)=(1,1)$ point of 'perfect convergence' can be reached because some of the decoding trajectories are curtailed owing to the limited interleaver length used.

${ }^{5}$ An open EXIT tunnel specifies the receive SNR value where there is a more widely open EXIT tunnel leading to the $(1,1)$ point and where decoding convergence to an infinitesimally low BER value can always be achieved, provided that the interleaver length is beyond a certain value and the number of iterations is sufficiently high [91]
}

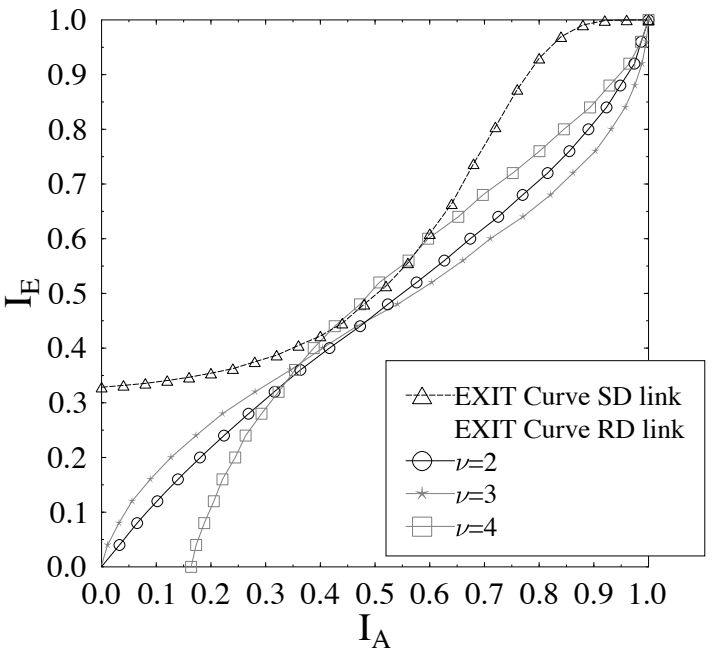

Fig. 22. The EXIT curves for the DSECCC-ID scheme for a $\mathrm{SNR}_{e}=$ $-3.5 \mathrm{~dB}$ both at the source as well as at the relay nodes. We portray the RD link's EXIT curves for three different values of $\nu$.

as well as that of the RSC decoder recorded at the RD link are plotted in Fig. 22. Since this EXIT-chart reflects the destination decoder's convergence after the completion of the SECCC iterations, only one of the pair of symmetric curves is shown. Our goal at this stage is to examine the extrinsic information exchange between the SECCC decoder and RSC decoder having different memory lengths. The RD link employs rates of $R_{3}=1 / 2, R_{4}=1 / 2$. As explicity shown in Fig. 22, we varied the memory of the RSC encoder in order to find the specific code memory, which has a low complexity, while simultaneoulsy matching the EXIT curve of the SECCC decoder of the SD link. It can be seen from Fig. 22 that the EXIT curves associated with $\nu=2$ and $\nu=3$ do not intersect the EXIT curve of the SD link when we have $\mathrm{SNR}_{e}=-3.5 \mathrm{~dB}$ at both the $\mathrm{SD}$ and at the RD link, while the $\nu=4$-curve does intersect it at the same SNR. Since $\nu=2$ represents a lower-complexity code, we opted for $\nu=2$ in our proposed scheme.

The EXIT curves and corresponding decoding trajectory are shown in Fig. 23. The number of iterations exchanging extrinsic information between the SECCC decoder of the SD link and the RSC decoder of the RD link is limited to $I_{s d, r d}=10$.

Step 3: The successful decoding convergence threshold of the DSECCC-ID system may be calculated with the aid of the EXIT curves, which intersect each other at $\mathrm{SNR}_{e}$ of $-3.65 \mathrm{~dB}$. Hence the staircase-shaped decoding trajectory will not reach the $(1,1)$ point of perfect convergence to a vanishingly low BER. But once the system is operating at $\mathrm{SNR}_{e}=-3.5 \mathrm{~dB}$ in the SD link and again at $\mathrm{SNR}_{e}=-3.5 \mathrm{~dB}$ in the $\mathrm{RD}$ link, an open tunnel emerges. Since $\mathrm{SNR}_{e}=-3.5 \mathrm{~dB}$ is higher than the threshold of $\mathrm{SNR}_{e}=-6.17 \mathrm{~dB}$, which guarantees an SECCC decoding convergence at the relay, the SR link may be deemed near-perfect. Another reason why we configure the system to operate an SNR higher than the minimum successful decoding SNR is because we want to have less 


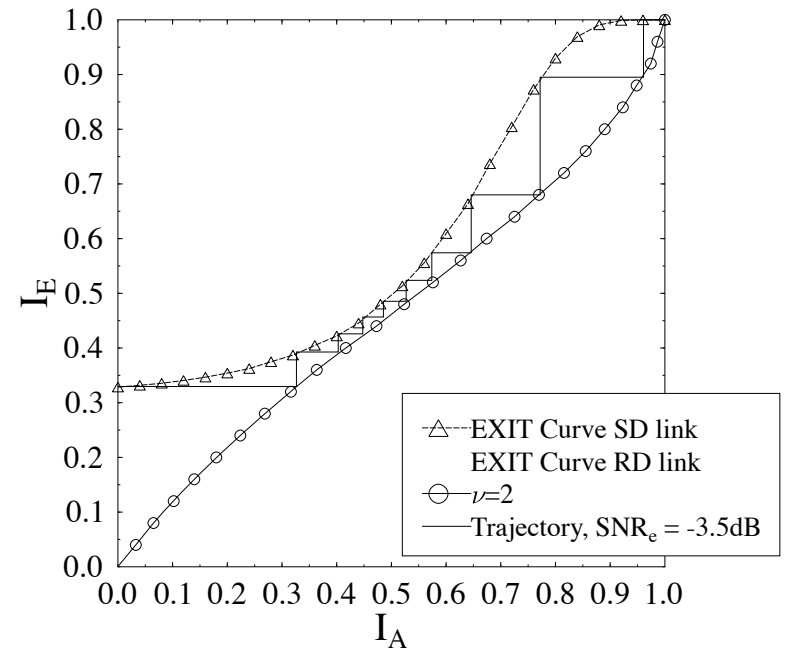

Fig. 23. The EXIT curves and a decoding trajectory of the DSECCC-ID scheme for $\mathrm{SNR}_{e}=-3.5 \mathrm{~dB}$ both at the source as well as at the relay nodes. The number of iterations exchanging extrinsic information between the SECCC and RSC decoders at the destination node is limited to $I_{s d, r d}=10$.

self-concatenated iterations at the SR link's receiver, namely $I_{s r}=8$ in this case.

The EXIT chart analysis is verified by computing the corresponding Monte-Carlo simulation based decoding trajectory for the DSECCC-ID scheme. The distinct decoding trajectory based on a frame length of 120000 bits is shown in Fig. 23 for an equivalent SNR of $-3.5 \mathrm{~dB}$ both at the source and at the relay. It matches the EXIT curves generated for the SD link, which employs the SECCC scheme and the RD link employing the RSC scheme, hence verifying the predicted results.

\section{E. Relay Capacity}

The two-hop half-duplex constrained relay-aided network capacity may be calculated by considering the capacity of the channel between the source, relay and the destination.

We first derive the upper and lower bounds on our halfduplex constrained relay-aided system's CCMC capacity as well as those of the DCMC capacity (constrained information rate) based on the approach proposed for full-duplex relay channels in [147]. The signals $X_{1}$ and $X_{2}$ are transmitted from the source $S$ seen in Fig. 18 during $T_{1}$ and $T_{2}$, respectively, while $Y_{1}$ and $Y_{2}$ represent the corresponding signals received at the destination $D$ of Fig. 18 during the consecutive time slots. Furthermore, $X$ and $Y$ are the transmitted and received signals at the relay $R$ of Fig. 18, respectively. The upper and lower bound on the CCMC and DCMC capacity of a halfduplex relay-aided system can then be derived by setting $X_{2}=$ 0 , because the source does not transmit in $T_{2}$. Consequently, the upper bound may be expressed as:

$$
\begin{aligned}
C_{\text {Coop }}^{U} \leq \max _{p\left(x_{1}, x\right)} \min \{ & \lambda E\left[I\left(X_{1} ; Y_{1}, Y\right)\right], \lambda E\left[I\left(X_{1} ; Y_{1}\right)\right]+ \\
& \left.(1-\lambda) E\left[I\left(X ; Y_{2}\right)\right]\right\}
\end{aligned}
$$

and the lower bound as:

$$
\begin{aligned}
C_{\text {Coop }}^{L} \geq \max _{p\left(x_{1}, x\right)} \min & \left\{\lambda E\left[I\left(X_{1} ; Y\right)\right], \lambda E\left[I\left(X_{1} ; Y_{1}\right)\right]+\right. \\
& \left.(1-\lambda) E\left[I\left(X ; Y_{2}\right)\right]\right\}
\end{aligned}
$$

where $I(A ; B)$ represents the mutual information for the channel having the i.u.d. input $A$ and the corresponding output $B$ for the case of CCMC capacity. By contrast, for the DCMC scenario the input $A$ is constituted by PSK/Quadrature Amplitude Modulated (QAM) symbols. Still referring to Equations 11 and $12, E($.$) denotes the expectation with respect to$ the fading coefficients, $p\left(x_{1}, x\right)$ represents the joint probability of the signals transmitted from the source and the relay, while $\lambda$ is the ratio of $T_{1}$ to the total frame duration, which is given by $\frac{N_{s}}{N_{s}+N_{r}}=\frac{3}{4}$. Similarly, we have $(1-\lambda)=\frac{N_{r}}{N_{s}+N_{r}}=$ $\frac{1}{4}$. The term $E\left[I\left(X_{1} ; Y_{1}, Y\right)\right]$ in Equation 11 represents the expected value of the mutual information between the signal transferred from the source node $S$ and the signals received at both the relay and destination nodes during $T_{1}$, while the term $E\left[I\left(X_{1} ; Y\right)\right]$ in Equation 12 considers the link spanning from the source node $S$ to the relay node $R$ in $T_{1}$. Furthermore, the term $E\left[I\left(X_{1} ; Y_{1}\right)\right]$ considers the transmission from the source node to the destination node in $T_{1}$. Finally, $E\left[I\left(X ; Y_{2}\right)\right]$ represents the expected value of the mutual information between the signals transferred from the relay node and the signal received at the destination node during $T_{2}$.

The corresponding constrained information rates of $E\left[I\left(X_{1} ; Y_{1}, Y\right)\right], E\left[I\left(X_{1} ; Y_{1}\right)\right], E\left[I\left(X ; Y_{2}\right)\right]$ and $E\left[I\left(X_{1} ; Y\right)\right]$ may be computed by using the Monte-Carlo averaging method [188]. Using Equations 11 and 12 we can calculate the DCMC and CCMC capacity of the two-hop relay-aided network, which is graphically shown in Fig. 24.

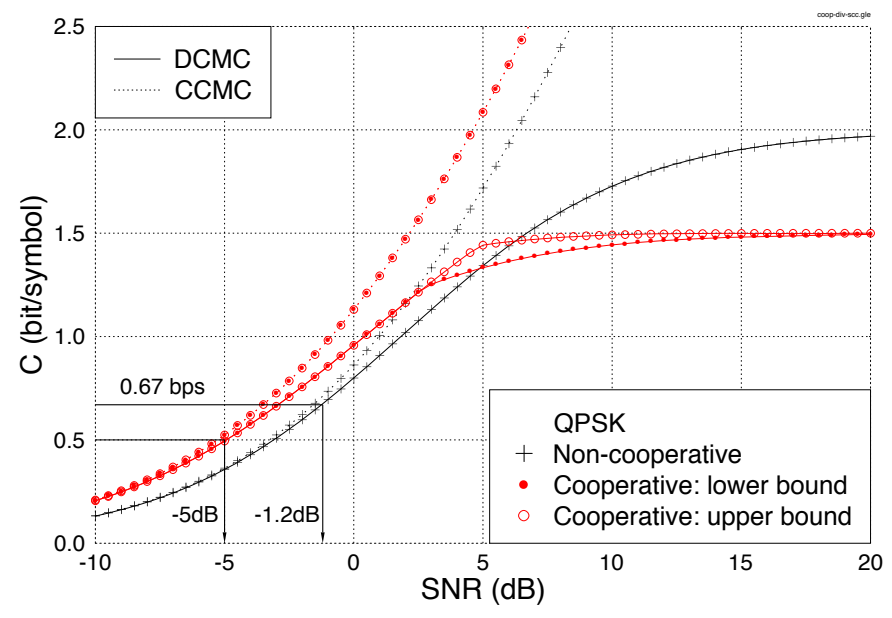

Fig. 24. The DCMC and CCMC capacity curves of cooperative network and non-cooperative QPSK assisted schemes. It can be seen that the DCMC of a cooperative network is $-5 \mathrm{~dB}$ at $0.5 \mathrm{bps}$. DCMC of a non-cooperative QPSK assisted scheme is $-1.20 \mathrm{~dB}$ at $0.67 \mathrm{bps}$.

\section{F. Results and Discussions}

Finally, we compare the achievable performance of the DSECCC-ID scheme employing a realistic relay node, which 


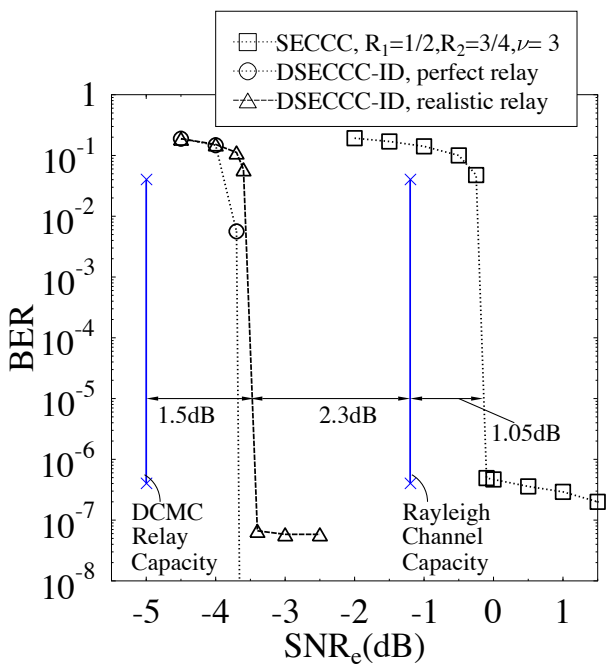

Fig. 25. BER versus equivalent SNR performance of the DSECCC-ID and SECCC schemes for a frame length of 120,000 bits. The DCMC relay-aided network capacity is $-0.5 \mathrm{~dB}$ at 0.5 bps as calculated from Fig. 24 .

potentially induces error propagation, to that of the noncooperative SECCC scheme. The BER versus equivalent SNR performance of the DSECCC-ID and SECCC schemes is shown in Fig. 25.

The SECCC scheme has a decoding threshold at $-0.2 \mathrm{~dB}$ and the tunnel at $-0.15 \mathrm{~dB}$. It performs $1.05 \mathrm{~dB}$ away from the Rayleigh fading channels's capacity calculated as $-1.20 \mathrm{~dB}$ from [2] and Fig. 24 at a BER of $10^{-5}$. The DSECCC-ID system has been analysed at $-3.5 \mathrm{~dB}$ stipulated at both the source and the relay employing the RSC encoder. Thus the DSECCC-ID outperforms the SECCC scheme by about $3.3 \mathrm{~dB}$ in SNR terms at a BER of $10^{-5}$, which corresponds to $3.3-$ $1.25=2.05 \mathrm{~dB}$ in terms of $E_{b} / N_{0}$.

As shown in Fig. 25, the proposed DSECCC-ID system is capable of performing within about $1.5 \mathrm{~dB}$ from the twohop relay-aided network's DCMC capacity of $-5 \mathrm{~dB}$ at $0.5 \mathrm{bps}$, as inferred from Fig. 24 at a BER of $10^{-5}$. By comparison, for the scheme proposed in [184], the signals arriving from the source and relay are superimposed at the destination, where a MAP detector and a turbo decoder of memory $\nu=4$ exchange extrinsic information, which were shown to be capable of achieving a BER of $10^{-5}$, at about $1.43 \mathrm{~dB}$ away from the capacity of the ergodic flat-fading channel at an overall effective throughput of $0.44 \mathrm{bps}$. We compare the two schemes' complexity by calculating the total number of trellis states, multiplied by the number of iterations at the corresponding decoders. This determines the number of Add-Compare-Select (ACS) arithmetic operations of a systolic array based silicon chip. The total complexity of our proposed decoder is estimated as follows.

The number of decoding iterations at the SR link's memory$\nu=3$ decoder are $I_{s r}=8$, therefore $I_{s r} \times 2^{\nu}=8 \times 8=64$ ACS operations are required at the relay. The SD link employs $I_{s d}=2$ iterations of a memory- 3 decoder, whereas the RD link employs a $\nu=2$ code. The number of iterations exchanging extrinsic information between the SECCC and RSC decoders at the destination node is limited to $I_{s d, r d}=10$.
Hence, the number of ACS operations at the destination is given by $I_{s d} \times 2^{3} \times 2^{2} \times I_{s d, r d}=640$. The overall number of ACS operations is therefore $64+640=704$.

For the case of [184] the turbo decoder used in the RD link employs 15 iterations between the two parallel concatenated turbo codes, while 15 iterations exchange extrinsic information between the MAP decoder and the turbo decoder of memory $\nu=4$ at the destination. Hence the total number of ACS operations required in [184] is $(15+15) \times 2 \times 2^{4}=960$. We note however that the complexity incurred by the MAP detector has not been included in the calculations. Hence our proposed system is capable of exhibiting a similar performance, while incurring a reduced overall complexity compared to the scheme advocated in [184].

\section{Conclusions, Design Guidelines and Future RESEARCH}

\section{A. Summary and Conclusions}

We have presented a suite of novel transceiver designs employing iteratively detected self-concatenated coding schemes in order to achieve a near-capacity performance, when operating in AWGN and Rayleigh fading channels. In order to eliminate the mismatch between the EXIT-chart and the MonteCarlo-simulation based decoding trajectory experienced in the context of the TCM based scheme discussed in [92], we proposed bit-based SECCCs. The mapper utilised Gray mapping, which mitigated the above-mentioned EXIT-chart mismatch. However, some information was lost, because the coded bits in each coded symbol are correlated. To recover this lost information, in Section III-B soft decision demapping was used. It was observed in Figure 13 that the SECCC-ID scheme of Figure 8 employing the SP demapper outperformed some of the GM based SECCC schemes. To analyse the exchange of extrinsic information between the SISO MAP decoder and the soft demapper of Figure 8, we employed 3-D EXIT charts in Figures 10 and 11. The accuracy of the 3-D EXIT chart based design was confirmed by the corresponding bit-by-bit Monte-Carlo BER simulations of Figure 13. Finally, in Section III-C we derived the union bound of SECCCs employing BPSK modulation for communication over both AWGN and uncorrelated Rayleigh fading channels, based on the novel uniform self-interleaver concept. In Section IV we proposed a power-efficient distributed scheme employing SECCCs for cooperative communications in order to mitigate the effects of large-scale shadow fading on the performance of wireless communication systems. The scheme is capable of providing substantial diversity-, throughput- as well as coding-gains for the case of a single-user scenario. Again, the novel threecomponent parallel concatenated decoder of Figure 20 was invoked. The proposed scheme was designed by conceiving the widely applicable design procedure of Section IV-D using EXIT charts. The related complexity analysis was carried out in Section IV-F and it was demonstrated that the proposed scheme has a low complexity. Despite the fact that the SR link was prone to decision errors, this simplified scheme was capable of approaching the DCMC capacity. 


\section{B. Design Guidelines}

- The first step in the design of FEC coding schemes in general and in SECCC and SECCC-ID coding schemes in particular is that of determining the code's specifications, such as the affordable decoding complexity expressed for example in terms of the number of ACS arithematic operations. This predetermines the resultant chip area versus decoding speed trade-offs, hence ultimately the maximum supported transmission rate.

- Another fundamental specification is the affordable delay, which determines the maximum tolerable interleaver length.

- Then the specific choice of the most appropriate SECCC component has to be resolved. As discussed in Section III, bit-based SECCC schemes designed with the aid of 2-D EXIT charts are accurate in predicting the convergence thresholds and they have flexible codingand puncturing-rates. Furthermore, more flexible threestage SECCC-ID schemes may be designed with the aid of 3-D EXIT charts. We demonstrated in Section III-C that in order to have a complete and accurate code design procedure the Truncated Union Bound (TUB) is necessary, which can be used to predict the error-floors, while EXIT charts may be invoked to predict the turbocliff-SNR in the design of near-capacity SECCCs.

- In the light of the inherent trade-off between the lowest possible turbo-cliff SNR and the lowest achievable residual error floor, we can use the EXIT-chart based codedesign procedure of Section III-B2 and the generator polynomials exemplified in Table IV to meet the dataintegrity requirements, such as the BER, SER or PER specifications.

- SECCCs provide the designer with a high degree of design-freedom, since they offer a vast range of options. These design options are exemplified by the type of component codes, their generator polynomials, code rate, puncturer schemes, interleaver designs and memory, bitto-symbol mapping schemes (such as Gray mapping, Anti-Gray mapping, Set-Partitioning), the choice of modulation schemes (such as coherent and non-coherent modems), irregular code designs, etc.

- When near-capacity operation is the over-riding design criterion, rather than that of minimizing the overall delay or complexity, the EXIT-chart-matching based designs of Section III suggest that 3 -stage concatenated designs may have to be invoked. This is, because they are capable of reducing the area of the open EXIT-tunnel and hence they facilitate decoding convergence to an infinitesimally low BER at near-capacity SNRs.

- Hence it is important to emphasize that maximizing the minimum distance of the code or directly searching for the code having the best distance profile or weightdistribution is no longer the most paramount design criterion. The EXIT-charts provide us with a more insightful tool for designing codes for near-capacity operation.

- When designing SECCCs for supporting wireless celledge users for example, the distributed code design principles of Section IV may be relied upon. More specif- ically, the distributed codes may move the constituent codes to separate relay nodes which have independently fading channels and hence provide a diversity gain. However, a powerful code is needed for all links of a relay-aided system, but especially for the SR link, in order to prevent error propagation. This suggests that the employment of a concatenated component code is of paramount importance at all nodes, particularly. Hence the coding scheme of the SR link has to be designed using EXIT charts, as detailed in Section IV-D. The propagation of decoding errors also has to be prevented along the RD link, which is achieved with the aid of another EXIT-chart matching procedure detailed in Section IV-D. Thus using DSECCC-ID schemes by employing a 3-stage decoder architecture, effectively reduces the potential error-floor often encountered in conventional 2-stage architectures. DSECCC-ID schemes impose a low complexity, where the ACS operations are distributed between the relay and destination nodes. Similarly to co-located constituent codes, it was demonstrated that EXIT charts are needed to design DSECCC-ID schemes using a widely applicable 3-step procedure:

- Decoding convergence threshold of the SECCC scheme of the SR link is calculated.

- EXIT curve of the SD link is matched against that of a suitable RD link EXIT curve.

- Convergence threshold of the DSECCC-ID scheme is then calculated. More explicity, by plotting the decoding trajectory of the DSECCC-ID scheme we can determine the number of iterations required between the SECCC and RSC decoders at the destination node in order to achieve perfect convergence to an infinitesimally low BER.

\section{Future Work}

Our future research will focus on designing reducedcomplexity SECCC-ID schemes. Furthermore, 3-D EXIT charts may also be used to design a SECCC-ID scheme concatenated with an outer codec, such as a video codec for enabling soft information exchange between the SECCC-ID decoder and the video decoder. Another area to explore is that of finding the union bound for various coding rates of the SECCCs combined with higher-order modulation schemes, using the uniform puncturing concept of Section III-C. Our future research will focus on enhancing the DSECCC-ID scheme of Section IV designed for cooperative communications in order to operate near the capacity, while imposing a low complexity using differential encoding and non-coherent detection, and dispensing with channel estimation [1], [6]. The next challenging issue will be that of reducing the total power, including the transmit power and the DSP-related power consumption in a relay-aided network. The question arises in a multi-hop network without line of sight propagation, as to how we can better utilize distributed coding in this cooperative network. Soft relaying has been proposed as a powerful method of combining the main advantages of both AAF and DAF signalling strategies. In [165], [170], [173] soft DAF has been shown to outperform the DAF and AAF 
signalling, where it was argued that the DAF signalling loses soft information and hence all operations were performed in the LLR domain. Similarly, another beneficial extension to consider is to create a hybrid of the DAF or AAF as the optimal relaying scheme according to the specific position of relays. If the available relay is closer to the source, then DAF gives a better performance, while if the relay is closer to the destination, then AAF is preferable [190]. Therefore, based on the performance improvements reported in the literature [190] while using soft information relaying, the DSECCCID scheme designed for a single user and the SPC-SECCCID conceived for two users will transmit soft estimates of the other users' data instead of performing hard decoding, since the hard-decoding solution would lose the advantage of soft information. Finally, the successive relaying principle of [191] may be invoked for mitigating the throughput loss imposed by the provision of the separate broadcast and cooperative phase. In order to avoid the complications of relay-synchronization, the asynchronous relaying regime of [192] may be invoked.

\section{REFERENCES}

[1] L. Hanzo, L.-L. Yang, E.-L. Kuan and K. Yen, Single and multi-carrier DS-CDMA: Multi-user detection, space-time spreading, synchronisation, networking and standards. Chichester, England: John Wiley and Sons Ltd and IEEE Press, 2003.

[2] L. Hanzo, S.X. Ng, T. Keller and W. Webb, Quadrature amplitude modulation: From basics to adaptive trellis-coded, turbo equalised and space-time coded OFDM, CDMA and MC-CDMA systems, 2nd Edition. Chichester, England: John Wiley and Sons Ltd and IEEE Press, 2004.

[3] C. E. Shannon, "A mathematical theory of communication," Bell Systems Technical Journal, vol. 27, pp. 379/623-423/656, July/October 1948.

[4] C. Berrou, A. Glavieux and P. Thitimajshima, "Near Shannon limit error-correcting coding and decoding: Turbo-codes (1)," in IEEE International Conference on Communications, vol. 2, Geneva, May 1993, pp. 1064-1070.

[5] L. Hanzo, T.H. Liew and B.L. Yeap, Turbo coding, turbo equalisation and space time coding for transmission over fading channels. Chichester, UK: Wiley: IEEE Press, 2002.

[6] L. Hanzo, O. Alamri, M. El-Hajjar and N. Wu, Near-capacity multifunctional MIMO systems. IEEE Press - John Wiley, April 2009.

[7] K.J.R. Liu, A.K. Sadek, W. Su and A. Kwasinski, Cooperative communications and networking. Cambridge University Press, 2009.

[8] M. Janani, A. Hedayat, T. Hunter and A. Nosratinia, "Coded cooperation in wireless communications: space-time transmission and iterative decoding," IEEE Trans. Signal Process., vol. 52, no. 2, pp. 362-371, February 2004.

[9] J. C. MacKay, Information theory, inference and learning algorithms. Cambridge University Press, 2003.

[10] R. Gallager, Low-density Parity-check Codes. Cambridge, MA: MIT Press, 1963.

[11] T.J. Richardson, A. Shokrollahi and R. Urbanke, "Design of capacityapproaching low-density parity-check codes," IEEE Trans. Inf. Theory, vol. 47, pp. 619-637, February 2001.

[12] R.W. Hamming, "Error detecting and error correcting codes," Bell Syst. Tech. Journal, vol. 29, pp. 41-56, 1950.

[13] I. Reed, "A class of multiple-error-correcting codes and the decoding scheme," IEEE Trans. Inf. Theory, vol. 4, no. 4, pp. 38-49, September 1954.

[14] D. E. Muller, "Application of boolean switching algebra to switching cicuit design," IEEE Trans. Comput., vol. 3, pp. 6-12, September 1954.

[15] P. Elias, "Coding for noisy channels," IRE Conv. Rept., pp. 37-47, 1955.

[16] E. Prange, "Cyclic error-correcting codes in two symbols," AFCRCTN-57, 103, Air Force Cambridge Research Center, Cambridge, Mass., 1972.

[17] A. Hocquenghem, "Codes correcteurs d'erreurs," Chiffres, vol. 2, pp. 147-156, 1959.
[18] R.C. Bose and D.K. Ray-Chaudhuri, "On a class of error correcting binary group codes," Information and Control, vol. 3, pp. 68-79, March 1960.

[19] I.S. Reed and G. Solomon, "Polynomial codes over certain finite fields," J. Soc. Ind. Appl. Math., vol. 8, pp. 300-304, June 1960.

[20] W.W. Peterson, "Encoding and error correction procedures for the Bose-Chaudhuri codes," IRE Trans. Inform. Theory, vol. IT-6, pp. 459470, September 1960.

[21] — Error-correcting codes. Cambridge, MA and New York MIT Press and Wiley, 1961.

[22] R. Gallager, "Low-density parity-check codes," IRE Transactions on Information Theory, vol. 8, no. 1, pp. 21-28, January 1962.

[23] R. Fano, "A heuristic discussion of probabilistic decoding," IEEE Trans. Inf. Theory, vol. 9, no. 2, pp. 64-74, April 1963.

[24] J.L. Massey, Threshold decoding. MIT Press: Cambridge, Mass., 1963.

[25] G. Forney, Concatenated codes. Cambridge: MIT Press, 1966.

[26] - -, "Generalized minimum distance decoding," IEEE Trans. Inf. Theory, vol. 12, no. 2, pp. 125-131, April 1966.

[27] E. Berlekamp, "Nonbinaty BCH decoding," Intl. Symp. on. Info. Th., 1967.

[28] L. D. Rudolph, "A class of majority logic decodable codes," IEEE Trans. Inf. Theory, vol. 13, pp. 305-307, May 1967.

[29] E. Berlekamp, Algebraic coding theory. McGraw-Hill, New York, 1968.

[30] R. Gallager, Information theory and reliable communication. New York: Wiley, 1968.

[31] F. Jelinek, "Fast sequential decoding algorithm using a stack," IBM J. Res. Develop., pp. 675-685, Nov 1969.

[32] J.L. Massey, "Shift-register synthesis and BCH decoding," IEEE Trans. Inf. Theory, vol. IT-15, pp. 122-127, 1969.

[33] A. Viterbi, "Convolutional codes and their performance in communication systems," IEEE Trans. Commun. [legacy, pre - 1988], vol. 19, pp. 751-772, October 1971.

[34] L. Bahl, C. Cullum, W. Frazer and F. Jelinek, "An efficient algorithm for computing free distance," IEEE Trans. Inf. Theory, vol. 18, no. 3, pp. 437-439, May 1972.

[35] D. Chase, "A class of algorithms for decoding block codes with channel measurement information," IEEE Trans. Inf. Theory, vol. IT-18, no. 1, pp. 170-182, January 1972.

[36] W.W. Peterson and E.J. Jr. Weldon, Error-correcting codes, 2nd ed. MIT Press: Cambridge, Mass., 1972.

[37] G.D. Jr. Forney, "The Viterbi algorithm," Proc. IEEE, vol. 61, no. 3, pp. 268-278, March 1973.

[38] L. Bahl, J. Cocke, F. Jelinek and J. Raviv, "Optimal decoding of linear codes for minimizing symbol error rate," IEEE Trans. Inf. Theory, vol. 20, no. 2, pp. 284-287, March 1974.

[39] Y. Sugiyama, M. Kasahara, S. Hirasawa and T. Namekawa, "A method for solving key equation for Goppa codes," Inf. and Control, vol. 27, pp. 87-99, 1975.

[40] F.J. MacWilliams and J.A. Sloane, The theory of error-correcting codes. North-Holland, Amsterdam, 1977.

[41] R. McEliece and L. Swanson, Reed-Solomon codes and their applications. IEEE Press, New York, 1994.

[42] J. Wolf, "Efficient maximum likelihood decoding of linear block codes using a trellis," IEEE Trans. Inf. Theory, vol. 24, no. 1, pp. 76-80, January 1978.

[43] V. Goppa, "Codes on algebraic curves," Soviet Math. Dokl., vol. 24, pp. 170-172, 1981.

[44] V.D. Goppa, Geometry and codes. Dordrecht [Netherlands] ; Boston : Kluwer Academic Publishers, 1988.

[45] G. Ungerböck, "Channel coding with multilevel/phase signals," IEEE Trans. Inf. Theory, vol. 28, no. 1, pp. 55-67, 1982.

[46] S. Lin and D.J. Jr. Costello, Error control coding: Fundamentals and applications. Englewood Cliffs, N.J.: Prentice-Hall, 1983.

[47] R.E. Blahut, Theory and practice of error control codes. Reading, MA, USA: Addison-Wesley, 1983.

[48] D. Divsalar and M.K. Simon, "Multiple trellis coded modulation (MTCM)," IEEE Trans. Commun., vol. 36, no. 4, pp. 410-419, April 1988.

[49] J. Hagenauer and P. Hoeher, "A Viterbi algorithm with soft-decision outputs and its applications," in IEEE Global Telecommunications Conference, GLOBECOM '89., Dallas, TX, November 1989, pp. 16801686.

[50] W. Koch and A. Baier, "Optimum and sub-optimum detection of coded data disturbed by time-varying intersymbol interference," in IEEE Global Telecommunications Conference, GLOBECOM '90., San Diego, CA, USA, December 1990, pp. 1679-1684. 
[51] E. Zehavi, "8-PSK trellis codes for a Rayleigh fading channel," IEEE Trans. Commun., vol. 40, pp. 873-883, May 1992.

[52] B. Honary, G. S. Markarian and P. G. Farrell, "Generalised array codes and their trellis structure," Electronics Letters, vol. 29, no. 6, pp. 541542, March 1993.

[53] B. Honary and G. Markarian, "Low-complexity trellis decoding of Hamming codes," Electronics Letters, vol. 29, no. 12, pp. 1114 -1116, June 1993.

[54] A.R. Hammons, P.V. Kumar, A.R. Calderbank, N.J.A. Sloane and P. Sole, "The $Z_{4}$-linearity of Kerdock, Preparata, Goethals and related codes," IEEE Trans. Inf. Theory, vol. 40, p. 301, 1994.

[55] J.A. Erfanian, S. Pasupathy and G. Gulak, "Reduced complexity symbol dectectors with parallel structures for ISI channels," IEEE Trans. Commun., vol. 42, pp. 1661-1671, 1994.

[56] MacKay and Neal, "Good codes based on very sparse matrices," in IMA: IMA Conference on Cryptography and Coding, LNCS lately (earlier: Cryptography and Coding II, Edited by Chris Mitchell, Clarendon Press, 1992), 1995.

[57] S.B. Wicker, Error control systems for digital communication and storage. Englewood Cliffs, NJ, USA: Prentice-Hall, 1994.

[58] P. Robertson, E. Villebrun and P. Hoeher, "A comparison of optimal and sub-optimal MAP decoding algorithms operating in the Log domain," in Proceedings of International Conference on Communications, Seattle, USA, June 1995, pp. 1009-1013.

[59] J. Hagenauer, E. Offer and L. Papke, "Iterative decoding of binary block and convolutional codes," IEEE Trans. Inf. Theory, vol. 42, no. 2, pp. 429-445, March 1996.

[60] V. Sidorenko, G. Markarian and B. Honary, "Minimal trellis design for linear codes based on the Shannon product," IEEE Trans. Inf. Theory, vol. 42, no. 6, pp. 2048-2053, November 1996.

[61] V. Tarokh, N. Seshadri and A. Calderbank, "Space-time codes for high data rate wireless communications: Performance criterion and code construction," in Proc IEEE International Conference on Communications '97, Montreal, Canada, 1997, pp. 299-303.

[62] H. Nickl, J. Hagenauer and F. Burkett, "Approaching Shannon's capacity limit by $0.27 \mathrm{~dB}$ using simple Hamming codes," IEEE Commun. Lett., vol. 1, no. 5, pp. 130-132, September 1997.

[63] C. Schlegel, Trellis coding. New York: John Wiley \& Sons, September 1997.

[64] X. Li and J.A. Ritcey, "Bit-interleaved coded modulation with iterative decoding," IEEE Commun. Lett., vol. 1, no. 6, pp. 169-171, November 1997.

[65] P. Robertson and T. Wörz, "Bandwidth-efficient turbo trellis-coded modulation using punctured component codes," IEEE J. Sel. Areas Commun., vol. 16, no. 2, pp. 206-218, February 1998.

[66] S.M. Alamouti, "A simple transmit diversity technique for wireless communications," IEEE J. Sel. Areas Commun., vol. 16, no. 8, pp. 1451-1458, 1998.

[67] V. Guruswami and M. Sudan, "Improved decoding of Reed-Solomon and algebraic-geometric codes," in IEEE Symposium on Foundations of Computer Science, 1998, pp. 28-39.

[68] X. Li and J.A. Ritcey, "Trellis-coded modulation with bit interleaving and iterative decoding," IEEE J. Sel. Areas Commun., vol. 17, no. 4, pp. 715-724, April 1999.

[69] S.M. Aji and R.J. McEliece, "The generalized distributive law," IEEE Trans. Inf. Theory, vol. 46, no. 2, pp. 325-343, March 2000.

[70] F.R. Kschischang, B.J. Frey and H.-A. Loeliger, "Factor graphs and the sum-product algorithm," IEEE Trans. Inf. Theory, vol. 47, no. 2, pp. 498-519, February 2001.

[71] J.G. Proakis, Digital communications, 4th edition ed. McGraw Hill Higher Education, December 1, 2000

[72] S. Siwamogsatham and M. Fitz, "Robust space-time coding for correlated Rayleigh fading channels," IEEE Trans. Signal Process., vol. 50, no. 10 , pp. 2408-2416, October 2002.

[73] H. Jafarkhani and N. Seshadri, "Super-orthogonal space-time trellis codes," IEEE Trans. Inf. Theory, vol. 49, no. 4, pp. 937-950, April 2003.

[74] R. Koetter and A. Vardy, "Algebraic soft-decision decoding of ReedSolomon codes," IEEE Trans. Inf. Theory, vol. 49, no. 11, pp. 28092825, November 2003.

[75] S. Lin and D.J. Jr. Costello, Error control coding: Second edition. Upper Saddle River, New Jersey: Prentice-Hall, 2004.

[76] T.K. Moon, Error correction coding: Mathematical methods and algorithms, 1st edition ed. John Wiley Sons, Inc., 2005.

[77] M.K. Simon and M.-S. Alouini, Digital communications over fading channels, 2nd edition ed. John Wiley Sons, Inc., 2005.
[78] A. Song, G. Wang and X.G. Xia, "Some super-orthogonal spacetime trellis codes based on non-PSK MTCM," IEEE Trans. Wireless Commun., vol. 4, no. 3, pp. 1214-1221, May 2005.

[79] E. Arıkan, "A Performance Comparison of Polar Codes and ReedMuller Codes," IEEE Commun. Lett., vol. 12, no. 6, pp. 447-449, June 2008.

[80] C. Berrou and A. Glavieux, "Near optimum error correcting coding and decoding: turbo-codes," IEEE Trans. Commun., vol. 44, no. 10, pp. 1261-1271, October 1996.

[81] S.L. Goff, A. Glavieux and C. Berrou, "Turbo-codes and high spectral efficiency modulation," in IEEE International Conference on Communications, New Orleans, LA, 1994, pp. 645-649.

[82] S. Benedetto, D. Divsalar, G. Montorsi and F. Pollara, "Bandwidth efficient parallel concatenated coding schemes," Electronics Letters, vol. 31, no. 24, pp. 2067-2069, November 1995.

[83] S.X. Ng, T.H. Liew, L-L. Yang and L. Hanzo, "Comparative study of TCM, TTCM, BICM and BICM-ID schemes," in IEEE Vehicular Technology Conference, Rhodes, Greece, May 2001, pp. 2450-2454.

[84] S. Benedetto, D. Divsalar, G. Montorsi and F. Pollara, "Serial concatenation of interleaved codes: performance analysis, design and iterative decoding," IEEE Trans. Inf. Theory, vol. 44, no. 3, pp. 909-926, May 1998.

[85] D. Divsalar and F. Pollara, "Serial and Hybrid Concatenated Codes with Applications," in International Symposium on Turbo Codes and Related Topics, Brest, France, September 1997.

[86] —- "Hybrid Concatenated Codes and Iterative Decoding," Jet Propulsion Laboratory, Pasadena, CA, TDA Progress Report 42-130, August 1997.

[87] S.X. Ng, M.F.U. Butt and L. Hanzo, "On the union bounds of selfconcatenated convolutional codes," IEEE Signal Process. Lett., vol. 16, no. 9, pp. 754-757, September 2009.

[88] S. Benedetto, D. Divsalar, G. Montorsi and F. Pollara, "Selfconcatenated trellis coded modulation with self-iterative decoding," in IEEE Global Telecommunications Conference, vol. 1, Sydney, NSW, Australia, 1998, pp. 585-591.

[89] H.-A. Loeliger, "New turbo-like codes," in IEEE International Symposium on Information Theory, Ulm, Jun/Jul 1997, p. 109.

[90] S. ten Brink, "Designing iterative decoding schemes with the extrinsic information transfer chart," AE $\ddot{U}$ International Journal of Electronics and Communications, vol. 54, no. 6, pp. 389-398, November 2000.

[91] — "Convergence behavior of iteratively decoded parallel concatenated codes," IEEE Trans. Commun., vol. 49, no. 10, pp. 1727-1737, October 2001.

[92] M.F.U. Butt, S.X. Ng and L. Hanzo, "EXIT chart aided design of near-capacity self-concatenated trellis coded modulation using iterative decoding," in 67th IEEE Vehicular Technology Conference, VTC-'08 Spring, Marina Bay, Singapore, May 2008, pp. 734-738.

[93] D. Divsalar and F. Pollara, "Multiple turbo codes for deep-space communications," Jet Propulsion Laboratory, Pasadena, CA, TDA Progress Report 42-121, May 1995.

[94] C. Douillard, M. Jezequel, C. Berrou, A. Picart, P. Didier and A. Glavieux, "Iterative correction of intersymbol interference: turbo equalization," European Transaction on Telecommunications, vol. 6, pp. 507-511, September/October 1995.

[95] S. Benedetto and G. Montorsi, "Iterative decoding of serially concatenated convolutional codes," Electronics Letters, vol. 32, no. 13, pp. 1186-1188, June 1996.

[96] S. Benedetto, D. Divsalar, G. Montorsi and F. Pollara, "A soft-input soft-output APP module for iterative decoding of concatenated codes," IEEE Communications Letters, vol. 1, no. 1, pp. 22-24, January 1997.

[97] G. Caire, G. Taricco and E. Biglieri, "Bit-interleaved coded modulation," in Proc. IEEE International Symposium on Information Theory (ISIT), Ulm, Germany, June/July 1997, p. 96.

[98] — " "Bit-interleaved coded modulation," IEEE Transactions on Information Theory, vol. 44, no. 3, pp. 927-946, May 1998.

[99] S. ten Brink, J. Speidel and R.-H. Yan, "Iterative demapping and decoding for multilevel modulation," in IEEE Global Telecommunications Conference (GLOBCOM), vol. 1, Sydney,NSW, 1998, pp. 579-584.

[100] X. Wang and H.V. Poor, "Iterative (turbo) soft interference cancellation and decoding for coded CDMA," IEEE Trans. Commun., vol. 47, no. 7, pp. 1046-1061, July 1999.

[101] O. F. Acikel and W. E. Ryan, "Punctured turbo-codes for BPSK/QPSK channels," IEEE Trans. Commun., vol. 47, no. 9, pp. 1315-1323, September 1999.

[102] D. Divsalar, S. Dolinar and F. Pollara, "Low complexity turbo-like codes," in 2nd International Symposium on Turbo Codes and Related Topics, Brest, France, September 2000, pp. 73-80. 
[103] _ _ "Serial concatenated trellis coded modulation with rate-1 inner code," in IEEE Global Telecommunications Conference (GLOBECOM), vol. 2, San Francisco, CA, 2000, pp. 777-782.

[104] I. Lee, "The effect of a precoder on serially concatenated coding systems with an ISI channel," IEEE Trans. Commun., vol. 49, no. 7, pp. 1168-1175, July 2001

[105] S. ten Brink, "Convergence of multidimensional iterative decoding schemes," in Conference Record of the Thirty-Fifth Asilomar Conference on Signals, Systems and Computers, vol. 1, Pacific Grove, CA, USA, 2001, pp. 270-274.

[106] H. El Gamal and A.R. Hammons, "Analyzing the turbo decoder using the Gaussian approximation," IEEE J. Sel. Areas Commun., vol. 47, pp. 671-686, February 2001.

[107] R. Ramamurthy and W.E. Ryan, "Convolutional double accumulate codes (or double turbo DPSK)," IEEE Commun. Lett., vol. 5, no. 4 pp. 157-159, April 2001.

[108] M. Tüchler and J. Hagenauer, "EXIT charts of irregular codes," in Conference on Information Science and Systems, Princtone, NJ, March 2002, pp. 748-753.

[109] M. Tüchler, S. ten Brink and J. Hagenauer, "Measures for tracing convergence of iterative decoding algorithms," in Proc. 4th International ITG Conference on Source and Channel Coding, Berlin, Germany, January 2002, pp. 53-60.

[110] M. Tüchler, "Convergence prediction for iterative decoding of threefold concatenated systems," in IEEE Global Telecommunications Conference (GLOBECOM ), vol. 2, Taipei, Taiwan, November 2002, pp. $1358-1362$.

[111] A. Sezgin, D. Wuebben and V. Kuehn, "Analysis of mapping strategies for turbo-coded space-time block codes," in Proc. IEEE Information Theory Workshop, Paris, France, March/April 2003, pp. 103-106.

[112] M. Tüchler, "Design of serially concatenated systems depending on the block length," IEEE Trans. Commun., vol. 52, no. 2, pp. 209-218, 2004.

[113] L. Lifang, D. Divsalar and S. Dolinar, "Iterative demodulation, demapping and decoding of coded non-square QAM," in IEEE Trans. Commun., vol. 53, January 2005, pp. 16-19.

[114] F. Brännström, L.K. Rasmussen and A.J. Grant, "Convergence analysis and optimal scheduling for multiple concatenated codes," IEEE Trans. Inf. Theory, vol. 51, no. 9, pp. 3354-3364, September 2005.

[115] Q. Luo and P. Sweeney, "Method to analyse convergence of turbo codes," Electronics Letters, vol. 41, no. 13, pp. 757 - 758, June 2005.

[116] C. Douillard and C. Berrou, "Turbo codes with rate- $\mathrm{m} /(\mathrm{m}+1)$ constituent convolutional codes," IEEE Trans. Commun., vol. 53, no. 10, pp. $1630-1638$, October 2005.

[117] I. Chatzigeorgiou, M.R.D. Rodrigues, I.J. Wassell and R. Carrasco, "A novel technique to evaluate the transfer function of punctured turbo codes," in IEEE International Conference on Communications, ICC 'O6., vol. 3, June 2006, pp. 1166-1171.

[118] W.R. Carson, I.J. Wassell and M. Rodrigues, "Optimal 8PSK mappings for BICM-ID over quasi-static fading channels," in 5th International Symposium on Turbo Codes and Related Topics, September 2008, pp. $128-133$

[119] S.X. Ng, O.R. Alamri, Y. Li, J. Kliewer and L. Hanzo, "Near-capacity turbo trellis coded modulation design based on EXIT charts and union bounds," IEEE Trans. Commun., vol. 56, no. 12, pp. 2030-2039, December 2008

[120] R.G. Maunder, J. Wang, S.X. Ng, L.-L. Yang and L. Hanzo, "On the performance and complexity of irregular variable length codes for near-capacity joint source and channel coding," IEEE Trans. Wireless Commun., vol. 7, no. 4, pp. 1338-1347, April 2008.

[121] C. Berrou, A. Graell i Amat, Y. Ould-Cheikh-Mouhamedou and Y. Saouter, "Improving the distance properties of turbo codes using a third component code: 3D turbo codes," IEEE Trans. Commun., vol. 57, no. 9, pp. $2505-2509$, September 2009.

[122] A. Grant, "Convergence of non-binary iterative decoding," in IEEE Global Telecommunications Conference, vol. 2, San Antonio, TX, USA, November 2001, pp. 1058-1062.

[123] H. Chen and A. Haimovich, "EXIT charts for turbo trellis-coded modulation," IEEE Commun. Lett., vol. 8, no. 11, pp. 668-670, November 2004.

[124] J. Kliewer, S.X. Ng and L. Hanzo, "Efficient computation of EXIT functions for non-binary iterative decoding," IEEE Trans. Commun., vol. 54, no. 12, pp. 2133-2136, December 2006.

[125] S. ten Brink, "Rate one-half code for approaching the Shannon limit by $0.1 \mathrm{~dB}$," Electronics Letters, vol. 36, no. 15, pp. 1293-1294, July 2000 .
[126] J. Hagenauer, "The EXIT chart - Introduction to extrinsic information transfer in iterative processing," in European Signal Processing Conference, Vienna, Austria, September 2004, pp. 1541-1548.

[127] S. Benedetto and G. Montorsi, "Unveiling turbo codes: Some results on parallel concatenated coding schemes," IEEE Trans. Inf. Theory, vol. 42, no. 2, pp. 409-428, March 1996.

[128] M.F.U. Butt, R.A. Riaz, S.X. Ng and L. Hanzo, "Near-capacity iteratively decoded binary self-concatenated code design using EXIT charts," in IEEE Global Communications Conference, GLOBECOM '08, New Orleans, USA, Nov/Dec 2008.

[129] J. Hagenauer, "Rate-compatible punctured convolutional codes (RCPC codes) and their applications," IEEE Trans. Commun., vol. 36, no. 4, pp. 389-400, April 1988.

[130] M.F.U. Butt, R.A. Riaz, S.X. Ng and L. Hanzo, "Near-capacity iterative decoding of binary self-concatenated codes using soft decision demapping and 3-D EXIT charts," IEEE Trans. Wireless Commun., vol. 9, no. 5, pp. 1608-1616, May 2010.

[131] B. Scanavino, G. Montorsi and S. Benedetto, "Convergence properties of iterative decoders working at bit and symbol level," in IEEE Global Telecommunications Conference, 2001. GLOBECOM '01., vol. 2, San Antonio, TX, USA, 2001, pp. 1037-1041.

[132] S.X. Ng, S. Das, J. Wang and L. Hanzo, "Near-capacity iteratively decoded space-time block coding," in 67th IEEE Vehicular Technology Conference, VTC-'08 Spring, Marina Bay, Singapore, May 2008.

[133] X. Li, A. Chindapol and J.A. Ritcey, "Bit-interleaved coded modulation with iterative decoding and 8PSK signaling," IEEE Trans. Commun., vol. 50, no. 8, pp. 1250-1257, August 2002.

[134] F. Schreckenbach, N. Görtz, J. Hagenauer and G. Bauch, "Optimization of symbol mappings for bit-interleaved coded Modulation with iterative decoding," IEEE Commun. Lett., vol. 7, no. 12, pp. 593-595, December 2003.

[135] N.H. Tran and H.H. Nguyen, "A novel multi-dimensional mapping of 8-PSK for BICM-ID," IEEE Trans. Wireless Commun., vol. 6, no. 3, pp. 1133-1142, March 2007.

[136] J. Wang, S. X. Ng, L. L. Yang and L. Hanzo, "Combined serially concatenated codes and MMSE equalization: An EXIT chart aided perspective," in IEEE 64th Vehicular Technology Conference, VTC'06 Fall, September 2006, pp. 1-5.

[137] S.X. Ng, J. Wang, M. Tao, L.-L. Yang and L. Hanzo, "Iteratively decoded variable-length space-time coded modulation: code construction and convergence analysis," IEEE Trans. Wireless Commun., vol. 6, no. 5, pp. 1953-1963, May 2007.

[138] D. Divsalar and M.K. Simon, "The design of trellis coded MPSK for fading channels: Set partitioning for optimum code design," IEEE Trans. Commun., vol. 36, pp. 1013-1021, September 1988.

[139] L.C. Perez, J. Seghers and D.J. Jr. Costello, "A distance spectrum interpretation of turbo codes," IEEE Trans. Inf. Theory, vol. 42, no. 6 , pp. 1698-1709, November 1996.

[140] Garello, R. and Pierleoni, P. and Benedetto, S., "Computing the free distance of turbo codes and serially concatenated codes with interleavers: algorithms and applications," IEEE J. Sel. Areas Commun., vol. 19 , no. 5, pp. 800-812, May 2001.

[141] Y. Li and B. Vucetic, "Optimization of space-time block codes based on multidimensional super-set partitioning," IEEE Signal Process. Lett., vol. 12, no. 4, pp. 317-320, April 2005.

[142] I. Chatzigeorgiou, M.R.D. Rodrigues, I.J. Wassell and R. Carrasco, "A union bound approximation for rapid performance evaluation of punctured turbo codes," in 41st Annual Conference on Information Sciences and Systems (CISS '07), March 2007, pp. 474-479.

[143] A. Graell i Amat, F. Brännström and L.K. Rasmussen, "On the design of rate-compatible serially concatenated convolutional codes," European Transactions on Telecommunications, vol. 18, no. 5, pp. 519527, 2007.

[144] M.F.U. Butt, R.A. Riaz, S.X. Ng and L. Hanzo, "Distributed SelfConcatenated Coding for Cooperative Communication," IEEE Trans. Veh. Technol., vol. 59, no. 6, pp. 3097-3104, July 2010.

[145] J. Abouei, H. Bagheri and A. Khandani, "An efficient adaptive distributed space-time coding scheme for cooperative relaying," IEEE Trans. Wireless Commun., vol. 8, no. 10, pp. 4957-4962, October 2009.

[146] J.N. Laneman, D.N. Tse and G.W. Wornell, "Cooperative diversity in wireless networks: Efficient protocols and outage behavior," IEEE Trans. Inf. Theory, vol. 50, no. 12, pp. 3062-3080, December 2004.

[147] T. Cover and A. El Gamal, "Capacity theorems for the relay channel," IEEE Trans. Inf. Theory, vol. 25, no. 5, pp. 572-584, 1979.

[148] G. Kramer, M. Gastpar and P. Gupta, "Cooperative strategies and capacity theorems for relay networks," IEEE Trans. Inf. Theory, vol. 51, no. 9, pp. 3037-3063, September 2005. 
[149] T.E. Hunter and A. Nosratinia, "Cooperation diversity through coding," in IEEE International Symposium on Information Theory, 2002.

[150] E. C. van der Meulen, "Three-terminal communication channels," Advanced Applied Probability, vol. 3, no. 1, pp. 120-154, 1971.

[151] A. Sendonaris, E. Erkip and B. Aazhang, "User cooperation diversity part I: System description," IEEE Trans. Commun., vol. 51, no. 11, pp. 1927-1938, 2003.

[152] E. Zimmermann, P. Herhold and G. Fettweis, "On the performance of cooperative relaying protocols in wireless networks," European Transactions on Telecommunications, vol. 16, no. 1, pp. 5-16, 2005.

[153] H. Ochiai, P. Mitran and V. Tarokh, "Design and analysis of collaborative diversity protocols for wireless sensor networks," in IEEE Vehicular Technology Conference, VTC-'04 Fall, Los Angeles, USA, September 2004, pp. 4645-4649.

[154] A. Sendonaris, E. Erkip and B. Aazhang, "Increasing uplink capacity via user cooperation diversity," in IEEE International Symposium on Information Theory, Cambridge, MA, August 1998.

[155] — , "User cooperation diversity part II: Implementation aspects and performance analysis," IEEE Trans. Commun., vol. 51, no. 11, pp. 1939-1948, 2003.

[156] M. Dohler, E. Lefranc and H. Aghvami, "Space-time block codes for virtual antenna arrays," in The 13th IEEE International Symposium on Personal, Indoor and Mobile Radio Communications, vol. 1, September 2002, pp. 414-417.

[157] J.N. Laneman and G.W. Wornell, "Distributed space-time-coded protocols for exploiting cooperative diversity in wireless networks," IEEE Trans. Inf. Theory, vol. 49, no. 10, pp. 2415-2425, 2003.

[158] P. Mitran, H. Ochiai and V. Tarokh, "Space-time diversity enhancements using collaborative communications," IEEE Trans. Inf. Theory, vol. 51, no. 6, pp. 2041-2057, June 2005.

[159] K. Azarian, H. El Gamal and P. Schniter, "On the achievable diversitymultiplexing tradeoff in half-duplex cooperative channels," IEEE Trans. Inf. Theory, vol. 51, no. 12, pp. 4152-4172, December 2005.

[160] A. Stefanov and E. Erkip, "Cooperative coding for wireless networks," IEEE Trans. Commun., vol. 52, no. 9, pp. 1470-1476, September 2004.

[161] E. C. van der Meulen, "A survey of multi-way channels in information theory: 1961-1976," IEEE Trans. Inf. Theory, vol. 23, no. 1, pp. 1-37, January 1977.

[162] M.C. Valenti and B. Zhao, "Distributed turbo codes: towards the capacity of the relay channel," in IEEE Vehicular Technology Conference, VTC-'03 Fall, vol. 1, October 2003, pp. 322-326.

[163] B. Zhao and M.C. Valenti, "Distributed turbo coded diversity for relay channel," IEE Electronics Letters, vol. 39, pp. 786-787, May 2003.

[164] R.U. Nabar, H. Bolcskei and F.W. Kneubuhler, "Fading relay channels: performance limits and space-time signal design," IEEE J. Sel. Areas Commun., vol. 22, no. 6, pp. 1099-1109, August 2004.

[165] H.H. Sneessens and L. Vandendorpe, "Soft decode and forward improves cooperative communications," in 6th IEE International Conference on 3G and Beyond, Washington, DC, November 2005, pp. 1-4.

[166] R. Hu and J. Li, "Exploiting Slepian-Wolf codes in wireless user cooperation," in IEEE 6th Workshop on Signal Processing Advances in Wireless Communications, June 2005, pp. 275-279.

[167] M. Yu and J. Li, "Is amplify-and-forward practically better than decode-and-forward or vice versa?" in IEEE International Conference on Acoustics, Speech and Signal Processing, vol. 3, March 2005.

[168] T.E. Hunter and A. Nosratinia, "Diversity through coded cooperation," IEEE Trans. Wireless Commun., vol. 5, no. 2, pp. 283-289, February 2006.

[169] T.E. Hunter, S. Sanayei and A. Nosratinia, "Outage analysis of coded cooperation," IEEE Trans. Inf. Theory, vol. 52, no. 2, pp. 375-391, February 2006.

[170] Y. Li, B. Vucetic, T.F. Wong and M. Dohler, "Distributed turbo coding with soft information relaying in multihop relay networks," IEEE $J$. Sel. Areas Commun., vol. 24, no. 11, pp. 2040-2050, November 2006.

[171] R. Hu and J. Li, "Practical compress-forward in user cooperation: Wyner-Ziv Cooperation," in IEEE International Symposium on Information Theory, Seattle, WA, July 2006, pp. 489-493.

[172] A. Host-Madsen, "Capacity bounds for cooperative diversity," IEEE Trans. Inf. Theory, vol. 52, no. 4, pp. 1522-1544, April 2006.

[173] T. Bui and J. Yuan, "A decode and forward cooperation scheme with soft relaying in wireless communication," in IEEE 8th Workshop on Signal Processing Advances in Wireless Communications (SPAWC), Helsinki, June 2007, pp. 1-5.

[174] M.N. Khormuji and E.G. Larsson, "Improving collaborative transmit diversity by using constellation rearrangement," in IEEE Wireless Communications and Networking Conference, Kowloon, March 2007, pp. $803-807$.
[175] X. Bao and J. Li, "Efficient message relaying for wireless user cooperation: Decode-Amplify-Forward (DAF) and Hybrid DAF and coded-cooperation," IEEE Trans. Wireless Commun., vol. 6, no. 11, pp. 3975-3984, November 2007.

[176] L. Xiao, T. Fuja, J. Kliewer and D. Costello, "A network coding approach to cooperative diversity," IEEE Trans. Inf. Theory, vol. 53, no. 10, pp. 3714-3722, October 2007.

[177] G. Yue, X. Wang, Z. Yang and A. Host-Madsen, "Coding schemes for user cooperation in low-power regimes," IEEE Trans. Signal Process., vol. 56, no. 5, pp. 2035-2049, May 2008.

[178] W. Zhang, Y. Li, X.-G. Xia, P.C. Ching and K.B. Letaief, "Distributed space-frequency coding for cooperative diversity in broadband wireless Ad Hoc networks," IEEE Trans. Wireless Commun., vol. 7, no. 3, pp. 995-1003, March 2008.

[179] T. Wang and G.B. Giannakis, "Complex field network coding for multiuser cooperative communications," IEEE J. Sel. Areas Commun., vol. 26, no. 3, pp. 561-571, April 2008.

[180] L. Badia, M. Levorato, F. Librino, and M. Zorzi, "Cooperation techniques for wireless systems from a networking perspective," IEEE Wireless Commun. Magazine, vol. 17, no. 2, pp. 89-96, April 2010.

[181] K. Tourki, M.-S. Alouini and L. Deneire, "Blind cooperative diversity using distributed space-time coding in block fading channels," IEEE Transactions on Communications, vol. 58, no. 8, pp. 2447-2456 , August 2010.

[182] F. Rossetto and M. Zorzi, "Mixing network coding and cooperation for reliable wireless communications," IEEE Wireless Communications, vol. 18, no. 1, pp. 15-21, February 2011.

[183] Y. Li, "Distributed coding for cooperative wireless networks: An overview and recent advances," IEEE Commun. Mag., vol. 47, no. 8, pp. 71-77, August 2009.

[184] Z. Zhang and T.M. Duman, "Capacity-approaching turbo coding for half-duplex relaying," IEEE Trans. Commun., vol. 55, no. 10, pp. 1895-1906, October 2007.

[185] J. Hu and T. M. Duman, "Low density parity check codes over wireless relay channels," IEEE Trans. Wireless Commun., vol. 6, no. 9, pp. 3384-3394, September 2007.

[186] M. Elfituri, W. Hamouda and A. Ghrayeb, "A convolutional-based distributed coded cooperation scheme for relay channels," IEEE Trans. Veh. Technol., vol. 58, no. 2, pp. 655-669, February 2009.

[187] A. Host-Madsen and J. Zhang, "Capacity bounds and power allocation for wireless relay channels," IEEE Trans. Inf. Theory, vol. 51, no. 6, pp. 2020-2040, June 2005.

[188] S.X. Ng and L. Hanzo, "On the MIMO channel capacity of multidimensional signal sets," IEEE Trans. Veh. Technol., vol. 55, no. 2, pp. 528-536, March 2006.

[189] S.X. Ng, J. Wang and L. Hanzo, "Unveiling near-capacity code design: The realization of Shannon's communication theory for MIMO channels," in IEEE International Conference on Communications, ICC '08, May 2008, pp. 1415-1419.

[190] L. Wang and L. Hanzo, "The resource-optimized differentially modulated Hybrid AF/DF cooperative cellular uplink using multiple-symbol differential sphere detection," IEEE Signal Process. Lett., vol. 16, no. 11 , pp. 965-968, November 2009.

[191] L. Kong, S. X. Ng, R. G. Maunder and L. Hanzo, "Successive Relaying Aided Near-Capacity Irregular Distributed Space-Time Coding," in IEEE Global Telecommunications Conference GLOBECOM, Honolulu, Hawaii, November 2009.

[192] S. Sugiura, S. Chen and L. Hanzo, "Distributed differential spacetime spreading for the asynchronous relay aided interference-free cooperative CDMA uplink," in IEEE International Conference on Communications, ICC '10, Cape Town, South Africa, May 2010, pp. $1-5$.

[193] P. Liu, Z. Tao, S. Narayanan, T. Korakis and S. Panwar, "CoopMAC: A cooperative MAC for wireless LANs," IEEE J. Sel. Areas Commun., vol. 25, no. 2, pp. 340-354, February 2007.

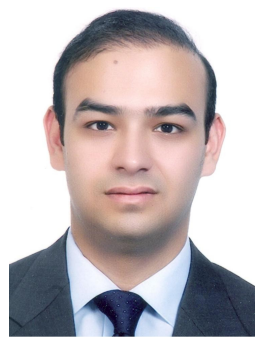

Muhammad Fasih Uddin Butt (S'08-M'10) received his B.E. degree from National University of Sciences \& Technology (NUST), Pakistan in 1999. He received his M.E. degree from Center for Advanced Studies in Engineering, UET Taxila, Pakistan with specialization in Digital Communication/Computer Networks in 2003 and his Ph.D. degree from Communications Research Group, School of Electronics and Computer Science, University 
of Southampton, U.K in June 2010. Since 2002 he has been serving as a faculty member in Department of Electrical Engineering, COMSATS Institute of Information Technology (CIIT), Islamabad, Pakistan. His research interests include Channel Coding, EXIT Chart Analysis and Distributed Coding for Cooperative Communications. He has published 15 research papers in IEEE journals and conference proceedings.

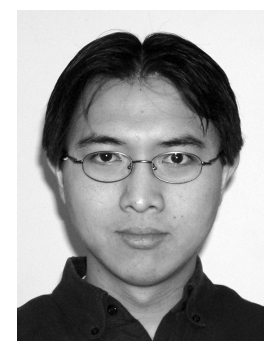

Soon Xin Ng (S'99-M'03-SM'08) received the B.Eng. degree (First class) in electronics engineering and the Ph.D. degree in wireless communications from the University of Southampton, Southampton, U.K., in 1999 and 2002, respectively. From 2003 to 2006, he was a postdoctoral research fellow working on collaborative European research projects known as SCOUT, NEWCOM and PHOENIX. Since August 2006, he has been a lecturer in wireless communications at the University of Southampton. He has been part of a team working on the OPTIMIX European project since March 2008. His research interests are mainly in adaptive coded modulation, channel coding, space-time coding, joint source and channel coding, OFDM, MIMO, cooperative communications and distributed coding. He has published numerous papers and coauthored a book in this field.

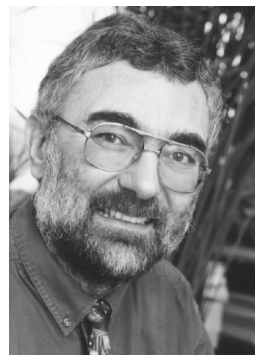

Lajos Hanzo (M'91-SM'92-F'04) FREng, FIEEE, FIET, received his degree in electronics in 1976 and his doctorate in 1983. In 2004 he was awarded the Doctor of Sciences (DSC) degree by the University of Southampton and in 2009 the honorary doctorate "Doctor Honaris Causa" by the Technical University of Budapest. During his 35-year career in telecommunications he has held various research and academic posts in Hungary, Germany and the UK. Since 1986 he has been with the School of Electronics and Computer Science, University of Southampton, UK, where he holds the chair in telecommunications. He has co-authored 20 John Wiley - IEEE Press books on mobile radio communications totalling in excess of 10,000 pages, published in excess of 1000 research papers and book chapters at IEEE Xplore, acted as TPC Chair of IEEE conferences, presented keynote lectures and been awarded a number of distinctions. Currently he is directing an academic research team, working on a range of research projects in the field of wireless multimedia communications sponsored by industry, the Engineering and Physical Sciences Research Council (EPSRC) UK, the European IST Programme and the Mobile Virtual Centre of Excellence (VCE), UK. He is an enthusiastic supporter of industrial and academic liaison and he offers a range of industrial courses. $\mathrm{He}$ is also an IEEE Distinguished Lecturer as well as a Governor of both the IEEE VTS. He is the Editor-in-Chief of the IEEE Press and a Chaired Prof. also at Tsinghua University, Beijing. For further information on research in progress and associated publications please refer to http://www-mobile.ecs.soton.ac.uk 